\title{
Calculating Heegaard-Floer Homology by Counting Lattice Points in Tetrahedra
}

\author{
Mahir Bilen Can $^{1}$ and Çağrı Karakurt ${ }^{2}$ \\ ${ }^{1}$ mcan@tulane.edu, Tulane and Yale Universities \\ ${ }^{2}$ karakurt@math.utexas.edu, University of Texas, Austin
}

October 30, 2018

\begin{abstract}
We introduce a notion of complexity for Sefiert homology spheres by establishing a correspondence between lattice point counting in tethrahedra and the Heegaard-Floer homology. This complexity turns out to be equivalent to a version of Casson invariant and it is monotone under a natural partial order on the set of Seifert homology spheres. Using this interpretation we prove that there are finitely many Seifert homology spheres with a prescribed HeegaardFloer homology. As an application, we characterize L-spaces and weakly elliptic manifolds among Seifert homology spheres. Also, we list all the Seifert homology spheres up to complexity two.
\end{abstract}

Keywords: Seifert homology sphere, Heegaard-Floer homology, rational and weakly elliptic singularities, L-space, numerical semigroup, tetrahedron

MSC-2010: 57R58, 57M27, 14J17, 05A15

\section{Introduction}

Heegaard-Floer homology, introduced by Ozsváth and Szabó in [14] and [13], is a prominent invariant for 3-manifolds. The goal of our article is to explore Heegaard-Floer homology from a combinatorial point of view in the special case of Seifert fibered homology spheres. Although it is 
more geometric than similar theories, such as Donaldson, or Seiberg-Witten theories, the definition of Heegaard-Floer homology involves a count of a certain moduli space of holomorphic disks into a symmetric product of a surface, which is in general a challenging analytical problem. On the other hand for a certain class of manifolds, namely plumbed manifolds with at most one bad vertex, works of Ozsváth and Szabó [12], and Nemethi [8] show that the calculation of Heegaard-Floer homology is a purely combinatorial problem. This class of manifolds is relatively small, but it is still large enough to include all Seifert fibered spaces (over $S^{2}$ ).

In [8], for a fixed plumbed 3-manifold, Nemethi finds an explicit algorithm whose output determines the Heegaard-Floer homology completely. An alternative algorithm is described in [12] by Ozsváth and Szabó. However, computing Heegaard-Floer homology for infinite families of 3-manifolds seems to be a formidable combinatorial problem for one has to determine all the local maxima and minima of infinite families of sequences which simultaneously solve an infinite family of non-homogeneous recurrence relations. See [9], [7], and [16] for some particular cases where this problem is handled.

To elaborate on the problem mentioned in the previous paragraph, let us briefly review Nemethi's method in the simplest case, where the 3-manifold is a Seifert homology sphere. To this end, let $p_{1}, \ldots, p_{l}$ be a list of pairwise relatively prime integers such that $1<p_{1}<p_{2}<\cdots<$ $p_{l}$. We denote by $\Sigma\left(p_{1}, p_{2}, \ldots, p_{l}\right)$ the Seifert fibered 3-manifold over $S^{2}$ with $l$ singular fibers whose Seifert invariants are given by $\left(e_{0},\left(p_{1}^{\prime}, p_{1}\right),\left(p_{2}^{\prime}, p_{2}\right), \ldots,\left(p_{l}^{\prime}, p_{l}\right)\right)$. Hence, $\left(x_{0}, x_{1}, \ldots, x_{l}\right)=$ $\left(e_{0}, p_{1}^{\prime}, p_{2}^{\prime}, \ldots, p_{l}^{\prime}\right)$ is the unique solution to the Diophantine equation

$$
x_{0} p_{1} p_{2} \cdots p_{l}+x_{1} p_{2} \cdots p_{l}+p_{1} x_{2} \cdots p_{l}+\cdots+p_{1} p_{2} \cdots x_{l}=-1,
$$

where $1 \leq x_{i} \leq p_{i}-1$, for $i=1,2, \ldots, l$. Equation (1.1) guarantees that $\Sigma\left(p_{1}, p_{2}, \ldots, p_{l}\right)$ has trivial first homology, so it is an integral homology sphere. To calculate Heegaard-Floer homology of $\Sigma\left(p_{1}, p_{2}, \ldots, p_{l}\right)$, we consider the sequence $\tau: \mathbb{N} \rightarrow \mathbb{Z}$ defined by the recurrence

$$
\tau(n+1)=\tau(n)+1+\left|e_{0}\right| n-\sum_{i=1}^{l}\left\lceil\frac{n p_{i}^{\prime}}{p_{i}}\right\rceil
$$

with the given initial condition $\tau(0)=0$. Here $\lceil y\rceil$ represents the minimum integer larger than $y$. We say that $\tau\left(n_{0}\right)$ is a local maximum of $\tau$, if there exist integers $a, b$ such that $a<n_{0}<b$ with $\tau(a)<\tau\left(n_{0}\right)>\tau(b)$, and $\tau$ is monotone increasing on the interval $\left[a, n_{0}\right]$ and monotone decreasing on $\left[n_{0}, b\right]$. Local minimum values of $\tau$ are defined similarly. It turns out that, up to a degree shift, the Heegaard-Floer homology is determined by the subsequence $\tau^{\prime}$ of $\tau$ consisting of all local minima and local maxima . 
In our first result we analyze the difference term in (1.2) in order to understand the local extrema of $\tau$. For notational convenience we focus our attention to Brieskorn spheres, which are by definition the Seifert homology spheres with three singular fibers $(l=3)$. Nevertheless, most of our arguments are adaptable for studying arbitrary number of singular fibers with some notational changes. See Theorem 4.1.

Theorem 1.3. Let $(p, q, r)$ be a triple of pairwise relatively prime integers with $1<p<q<r$. Define $\Delta: \mathbb{N} \rightarrow \mathbb{Z}$

$$
\Delta(n)=1+\left|e_{0}\right| n-\left\lceil\frac{n p^{\prime}}{p}\right\rceil-\left\lceil\frac{n q^{\prime}}{q}\right\rceil-\left\lceil\frac{n r^{\prime}}{r}\right\rceil,
$$

where $\left(e_{0}, p^{\prime}, q^{\prime}, r^{\prime}\right)$ is defined by

$$
e_{0} p q r+p^{\prime} q r+p q^{\prime} r+p q r^{\prime}=-1
$$

with $0 \leq p^{\prime} \leq p-1,0 \leq q^{\prime} \leq q-1,0 \leq r^{\prime} \leq r-1$. Define the constant

$$
N_{0}=p q r-p q-q r-p r .
$$

Suppose $(p, q, r) \neq(2,3,5)$. Then the following holds.

1. $N_{0}$ is a positive integer.

2. $\Delta(n) \geq 0$, for all $n>N_{0}$.

3. $\Delta(n)=-\Delta\left(N_{0}-n\right)$, for all $n$ with $0 \leq n \leq N_{0}$.

4. $\Delta(n) \in\{-1,0,1\}$, for all $n$ with $0 \leq n \leq N_{0}$.

5. For $0 \leq n \leq N_{0}$, one has $\Delta(n)=1$ if and only if $n$ is an element of the numerical semigroup $G(p q, p r, q r)$ minimally generated by $p q$, qr, and pr. (We consider 0 as an element of the semigroup, hence it is always true that $\Delta(0)=1$.)

If $(p, q, r)=(2,3,5)$, then $\Delta(n) \geq 0$ for all $n \in \mathbb{N}$.

As we justify later, the above theorem provides us with a fast and practical means for calculation of the Heegaard-Floer homology of a Brieskorn sphere. More importantly it gives a partial answer to the realization problem which we explain now.

Let $U$ be a formal variable and $Y$ be a closed, oriented 3-manifold. The Heegaard-Floer homology of $Y$ is a $\mathbb{Z}_{2}$-graded $\mathbb{Z}[U]$-module with a decomposition $H^{+}(Y) \simeq \oplus_{\mathfrak{s}} H F^{+}(Y, \mathfrak{s})$ into $\mathbb{Z}_{2}$-graded 
$\mathbb{Z}[U]$-submodules indexed by the $\operatorname{Spin}^{c}$ structures on $Y$. In the case of integral homology spheres the decomposition simplifies; there is unique $\operatorname{Spin}^{c}$ structure, and the $\mathbb{Z}_{2}$ grading lifts to a $\mathbb{Z}$-grading such that $U$ has degree -2 . Therefore, the following question becomes natural:

Question 1.4. Which $\mathbb{Z}$-graded $\mathbb{Z}[U]$-module can be realized as the Heegaard-Floer homology of a Seifert homology sphere $Y$ ?

It is known for integral homology spheres that $H F^{+}$decomposes into $\mathbb{Z}[U]$-submodules as follows

$$
H F^{+}(Y) \simeq \mathcal{T}_{(d)}^{+} \oplus H F_{\text {red }}(Y)
$$

where $\mathcal{T}_{(d)}^{+}$is a copy of $\mathbb{Z}\left[U, U^{-1}\right] / U \cdot \mathbb{Z}[U]$ on which we impose a grading so that the minimal degree is $d$. Furthermore, $H F_{\text {red }}(Y)$ is finitely generated. If $Y$ is a Seifert homology sphere oriented so that it bounds a positive definite plumbing, then $\mathrm{HF}^{+}(Y)$ is supported at even degrees only.

Let us illustrate how Theorem 1.3 is useful in the processes of solving Question 1.4. Recall that a 3-manifold $Y$ is said to be an $L$-space, if it is a rational homology sphere, and $H F_{\text {red }}(Y, \mathfrak{s})=0$ for every $\operatorname{Spin}^{c}$ structure $\mathfrak{s},[11]$.

Conjecture 1.5. If an irreducible integral homology sphere $Y$ is an $L$-space, then $Y$ is either the 3 -sphere, or the Poincaré homology sphere $\Sigma(2,3,5)$ with either orientation.

This conjecture is verified for Seifert homology spheres independently by Rustamov [15] and Eftekhary [4]. There is also an implicit proof of the same statement when one combines the results of [3] with [6]. Here we give an alternative, elementary proof.

Theorem 1.6. ([15], [4]) If a Seifert homology sphere $Y$ is an L-space, then $Y$ is homeomorphic to $S^{3}$ or $\pm \Sigma(2,3,5)$.

Among Seifert manifolds, $L$-spaces are precisely those 3-manifolds with $\Delta(n) \geq 0$ for all $n \geq \mathbb{N}$. Therefore, Theorem 1.3 is sufficient to prove Theorem 1.6 in the case of three singular fibers. The case of arbitrarily many singular fibers follows from an extension of our theorem to that setting.

Above results suggest that the sum of negative values of the $\Delta$ function is a significant quantity for it defines a kind of "complexity" for the Heegaard-Floer homology. Indeed, what we observe above is that the complexity 0 Seifert manifolds are precisely the $L$-spaces. Therefore, our next definition is meaningful. 
Definition 1.7. Let $p_{1}, \ldots, p_{l}$ be a list of relatively prime integers such that $1<p_{1}<\cdots<p_{l}$. For $\tau(n)$ as defined in (1.2), put $\Delta(n)=\tau(n+1)-\tau(n)$. We define

$$
\kappa\left(p_{1}, p_{2}, \ldots, p_{l}\right)=\left|\sum_{i=0}^{\infty} \min \{0, \Delta(n)\}\right| .
$$

It follows from [8] that if two Heegaard-Floer homology groups $\operatorname{HF}^{+}\left(\Sigma\left(p_{1}, p_{2}, \ldots, p_{l}\right)\right)$ and $H F^{+}\left(\Sigma\left(q_{1}, q_{2}, \ldots, q_{l}\right)\right)$ are isomorphic, then the corresponding kappa invariants $\kappa\left(p_{1}, p_{2}, \ldots, p_{l}\right)$ and $\kappa\left(q_{1}, q_{2}, \ldots, q_{l}\right)$ are equal. The converse does not hold in general, however, as we show, there are only finitely many isomorphism types of $\mathbb{Z}[U]$-modules which might appear as the HeegaardFloer homology of a Seifert homology sphere with a prescribed $\kappa$.

Theorem 1.8. For any positive integer $k$, there exists finitely many tuples $\left(p_{1}, p_{2}, \ldots, p_{l}\right)$ such that $\kappa\left(p_{1}, p_{2}, \ldots, p_{l}\right)=k$, where $p_{1}, p_{2}, \ldots, p_{l}$ are pairwise relatively prime, and $1<p_{1}<p_{2}<$ $\cdots<p_{l}$. Consequently, there exist at most finitely many Seifert homology spheres with prescribed Heegaard-Floer homology.

By contrast, we should mention that it is possible to find many infinite families of irreducible integral homology spheres with isomorphic Heegaard-Floer homology. See, for example, Proposition 1.2 of [1].

The most important ingredient in the proof of Theorem 1.8 is the monotonicity property of $\kappa$ with respect to a partial ordering on the set of tuples. We prove this property in propositions 3.12, 4.13, and 4.14. These results together with sufficient computational power, allows one to list all the graded $\mathbb{Z}[U]$-modules that could appear as the Heegaard-Floer homology of a Seifert homology sphere up to a given complexity. In the following theorem, we give this list up to $\kappa=2$.

Theorem 1.9. Table 1 contains the list of all graded $\mathbb{Z}[U]$-modules that are isomorphic to a Heegaard-Floer homology for some Seifert homology sphere with $\kappa \leq 2$. Additionally, for each such $\mathbb{Z}[U]$-module $M$, the table contains the list of all Seifert homology spheres whose HeegaardFloer homology is $M$.

Note that Theorem 1.6 is a special case of Theorem 1.9 for which $\kappa=0$. We should note also that the $\mathrm{HF}^{+}(-Y)$ completely determines the Heegaard-Floer homology of positively oriented $Y$, so there is no loss of information in Theorem 1.9.

Using a relation between the Euler characteristic of Heegaard-Floer homology and the Casson invariant, we relate $\kappa$ to other well known invariants of Seifert homology spheres. Recall that 


\begin{tabular}{|c|c|c|c|}
\hline Brieskorn Sphere $Y$ & $\kappa$ & $d(-Y)$ & $H F^{+}(-Y)$ \\
\hline$S^{3}$ & 0 & 0 & $\mathcal{T}_{(0)}^{+}$ \\
\hline$\Sigma(2,3,5)$ & 0 & -2 & $\mathcal{T}_{(-2)}^{+}$ \\
\hline$\Sigma(2,3,7)$ & 1 & 0 & $\mathcal{T}_{(0)}^{+} \oplus \mathbb{Z}_{(0)}$ \\
\hline$\Sigma(2,3,11)$ & 1 & -2 & $\mathcal{T}_{(-2)}^{+} \oplus \mathbb{Z}_{(-2)}$ \\
\hline$\Sigma(2,3,13), \Sigma(2,5,7), \Sigma(3,4,5)$ & 2 & 0 & $\mathcal{T}_{(0)}^{+} \oplus \mathbb{Z}_{(0)} \oplus \mathbb{Z}_{(0)}$ \\
\hline$\Sigma(2,3,17), \Sigma(2,5,9)$ & 2 & -2 & $\mathcal{T}_{(-2)}^{+} \oplus \mathbb{Z}_{(-2)} \oplus \mathbb{Z}_{(-2)}$ \\
\hline
\end{tabular}

Table 1: Seifert homology spheres with $\kappa \leq 2$.

every Seifert fibered space is the boundary of a 4-manifold that is plumbing of disk bundles over spheres, where the plumbing is done according to a negative definite star shaped weighted tree. Such a plumbing configuration is unique up to blow-up and blow-down. Suppose we fix one such plumbing and let $s$ denote the number of its vertices. Also, let $K$ denote its canonical cohomology class. Then the number $K^{2}+s$ is invariant under blow-up and blow-down, so it defines an invariant of the Seifert fibered space.

Proposition 1.10. For a Seifert homology sphere $Y=\Sigma\left(p_{1}, p_{2}, \ldots, p_{l}\right)$, the following equality holds

$$
\kappa\left(p_{1}, p_{2}, \ldots, p_{l}\right)=\lambda(-Y)-\left(K^{2}+s\right) / 8,
$$

where $\lambda(-Y)$ is the Casson invariant of $-Y$ [2], normalized so that the Poincaré homology sphere $\Sigma(2,3,5)$ oriented as the boundary of negative $E_{8}$ plumbing satisfies $\lambda(-\Sigma(2,3,5))=-1$.

In our next result we observe a remarkable connection between $\kappa$, numerical semigroups and lattice points in tetrahedra. Casson invariants of Brieskorn spheres have similar interpretations. See [5]. We state it for the special case of 3-singular fibers here. There is also a more technical statement that works for arbitrary number of singular fibers which we state in Theorem 4.5.

Theorem 1.11. Given a Brieskorn sphere $\Sigma(p, q, r)$ with defining integers $1<p<q<r$, its kappa invariant $\kappa(p, q, r)$ is equal to the number of lattice points inside the tetrahedron with vertices $(0,0,0),\left(N_{0} / p q, 0,0\right),\left(0, N_{0} / p r, 0\right)$, and $\left(0,0, N_{0} / q r\right)$, where $N_{0}=p q r-p q-p r-q r$. In other words, $\kappa(p, q, r)$ equals the cardinality of the set $G \cap\left[0, N_{0}\right]$, where $G=G(p q, p r, q r)$ is the numerical semigroup generated by $0, p q, q r$, and $p r$.

We push our techniques further to study a class of Brieskorn spheres that has a simple HeegaardFloer homology. The following definition is due to Nemethi [8]. 
Definition 1.12. A rational homology sphere $Y$ which is the boundary of a negative definite plumbing tree with at most one bad vertex is said to be weakly elliptic, if its Heegaard-Floer homology in the canonical Spin ${ }^{c}$ structure is of the form $T_{(d)}^{+} \oplus\left(\mathbb{Z}_{(d)}\right)^{l}$ for some $l \geq 1$ and some even integer $d$.

It is shown in Proposition 6.5 of [8] that, if $Y$ weakly elliptic, then it is the link of a weakly elliptic singularity. Our next result gives the complete list of weakly elliptic Seifert homology spheres.

Theorem 1.13. A Brieskorn sphere $\Sigma=\Sigma(p, q, r)$ is weakly elliptic if and only if $(p, q, r)$ is equal to one of the following triplets; $(3,4,5),(2,5,7),(2,5,9)$, or $(2,3, r)$ with $\operatorname{gcd}(6, r)=1$ and $r>5$. There are no weakly elliptic Seifert homology spheres with more than three singular fibers.

To further analyze the relationship between lattice point counting and $\tau$ of $\Sigma\left(p_{1}, \ldots, p_{l}\right)$, we compare their generating functions. Our computations show that the generating function of $\tau(n)$ is a rational function, similar to the generating function of the sequence counting the lattice points on the hyperplane $p_{1} x_{1}+p_{2} x_{2}+\cdots+p_{l} x_{l}=n$ that lie in the first orthant of $\mathbb{R}^{l}$. For the sake of space, here we write only the simplified version of the generating function of $\tau(n)$. See Theorem 7.2 for its explicit form.

Theorem 1.14. The generating function $F(x)=\sum_{n} \tau(n) x^{n}$ is given by

$$
F(x)=\frac{G(x)}{\left(1-x^{p_{1}}\right)\left(1-x^{p_{2}}\right) \cdots\left(1-x^{p_{l}}\right)},
$$

where $G(x)$ is a polynomial in $x$ with degree less than or equal to $p_{1}+p_{2}+\cdots+p_{l}-1$. Furthermore, if $\left|e_{0}\right| \neq 1$, then the degree of $G(x)$ is exactly $p_{1}+p_{2}+\cdots+p_{l}-1$.

The organization of our paper is as follows. In Section 2 we review Nemethi's method and see how Heegaard-Floer homology is calculated from the $\tau$-function. We analyze the $\Delta$-function in Section 3, and prove therein Theorem 1.3 and Theorem 1.11. We extend our results to arbitrary number of singular fibers in Section 4. Theorems 1.6, 1.8, and 1.9 are proved in Section 5. In Section 6, we characterize weakly elliptic Brieskorn spheres interms of certain numerical semigroups and prove Theorem 1.13. Finally, we conclude our paper by calculating the generating function of $\tau(n)$ in Section 7. 


\section{Graded Roots and Heegaard-Floer Homology}

Here we review the definition of a "graded root," and discuss its basic properties. For more information and background, we recommend [8] and Section 2 of [1].

Definition 2.1. A graded root is a pair $(R, \chi)$, where $R$ is an infinite tree, and $\chi$ is an integer valued function defined on the vertex set $V=V(R)$ of $R$ satisfying the following properties.

1. $\chi(u)-\chi(v)= \pm 1$, if there is an edge connecting $u$ and $v$.

2. $\chi(u)>\min \{v, w\}$, if there are edges connecting $u$ to $v$, and $u$ to $w$.

3. $\chi$ is bounded below.

4. $\chi^{-1}(k)$ is finite for every $k$.

5. $\left|\chi^{-1}(k)\right|=1$ for $k$ large enough.

In Figure 1 we give an example of a graded root, where the infinite tree $R$ is drawn on left, and the function $\chi$ is obtained from the heights of the vertices.

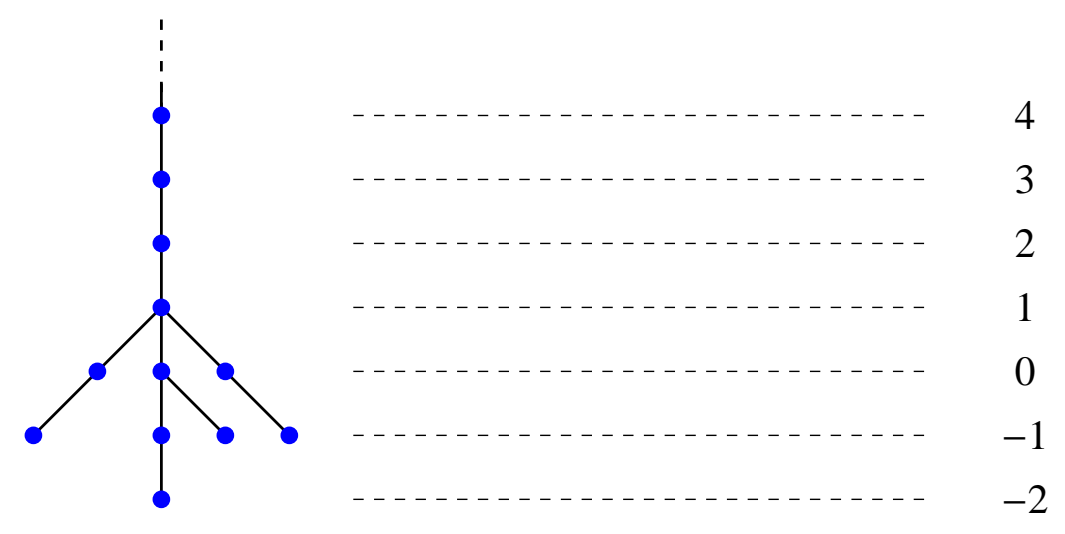

Figure 1: A graded root.

The branches of $R$ are enumerated from left to right, and the vertex at the bottom of the $i$-th branch is called the $i$-th vertex. Let us denote by $a_{i}$ the value of $\chi$ on the $i$-th vertex, and denote by $b_{i}$ the value of $\chi$ at the branching vertex connecting $i$-th branch to the $(i+1)$-th branch. Then the data of $(R, \chi)$ is encoded in the sequence $\left[a_{1}, b_{1}, a_{2}, b_{2}, \ldots, a_{n-1}, b_{n-1}, a_{n}\right]$. For example, the graded root given in Figure 1 is represented by the sequence $[-1,1,-2,0,-1,1-1]$. 
Conversely, any sequence $\left[a_{1}, b_{1}, a_{2}, b_{2}, \ldots, a_{n-1}, b_{n-1}, a_{n}\right]$ satisfying $b_{i}>\max \left\{a_{i}, a_{i+1}\right\}$, for $i=$ $1, \ldots, n-1$ determines a graded root. For a given sequence $\tau$ with this property, we denote the corresponding graded root by $\left(R_{\tau}, \chi_{\tau}\right)$.

The nomenclature of "graded root" is explained by the natural correspondence between graded roots and graded $\mathbb{Z}[U]$-modules. Consider the free $\mathbb{Z}$-module generated by the vertex set $V$. The $U$ action is described as follows. For a given vertex $v, U \cdot v$ has a summand supported at the vertex $w$, if there is an edge connecting $v$ to $w$, and $\chi(v)>\chi(w)$. Then the action of $U$ is extended by linearity. Finally, the grading is determined by the requirement that every vertex $v$ has degree $2 \chi(v)$. Given a graded root $(R, \chi)$, we denote the associated $\mathbb{Z}[U]$-module by $\mathbb{H}(R, \chi)$. For example, for the graded root given in Figure 1, the associated $\mathbb{Z}[U]$-module is isomorphic to $\mathcal{T}_{(-4)}^{+} \oplus \mathcal{T}_{(-2)}^{1} \oplus \mathcal{T}_{(-2)}^{1} \oplus \mathbb{Z}_{(-2)}$. Here, we use the following notation: $\mathcal{T}_{(d)}^{+}=\mathbb{Z}\left[U, U^{-1}\right] /\langle U\rangle$, and $\mathcal{T}_{(d)}^{n}=\mathbb{Z}[U] /\left\langle U^{n+1}\right\rangle$; both groups are graded so that $U$ has degree -2 , and the minimal degree is $d$.

Fix a Seifert homology sphere $\Sigma\left(p_{1}, \ldots, p_{l}\right)$, and let $\tau$ denote the sequence defined recursively as in (1.2). It is known that $\tau(n)$ is an increasing function of $n$, for all sufficiently large $n \gg 0$. It follows that the subsequence consisting of local minima and local maxima of $\tau$ is a finite sequence. By abuse of notation, we denote this finite subsequence by $\tau$, also. Now consider the graded root given by $\tau$ and its $\mathbb{Z}[U]$-module $\mathbb{H}\left(R_{\tau}, \chi_{\tau}\right)$. It turns out that, up to a global degree shift the HeegaardFloer homology of $\Sigma\left(p_{1}, \ldots, p_{l}\right)$ is isomorphic to $\mathbb{H}\left(R_{\tau}, \chi_{\tau}\right)$.

The degree shift is calculated as follows. Let $X$ denote the 4-manifold $X$ bounding $\Sigma\left(p_{1}, \ldots, p_{l}\right)$, which is a star shaped plumbing of certain disk bundles over 2-sphere with a negative definite intersection form. The second homology of $X$ has a natural basis $e_{0}, e_{1}, \ldots, e_{s-1}$ consisting of base spheres. Here, $e_{0}$ corresponds to the central vertex in the plumbing graph, and $s$ is equal to the total number of vertices. The canonical 2-cohomology class $K$ is defined by the requirement that $K\left(e_{i}\right)=-e_{i} \cdot e_{i}-2$. Then, the desired degree shift is given by $-\left(K^{2}+s\right) / 4$.

An alternative approach utilizes the "Dedekind sums" for computing the degree shift. The Dedekind sum, $s(p, q)$ is calculated recursively by setting $s(1,1)=0$ and repeatedly applying the reciprocity law

$$
s(p, q)+s(q, p)=-\frac{1}{4}+\frac{1}{12}\left(\frac{p}{q}+\frac{q}{p}+\frac{1}{p q}\right),
$$

and using the rule stating that whenever $r \equiv p \bmod q$, the equality $s(p, q)=s(r, q)$ holds.

It is shown in [10] that

$$
K^{2}+s=\epsilon^{2} e+e+5-12 \sum_{i=1}^{l} s\left(p_{i}^{\prime}, p_{i}\right),
$$


where

$$
e=e_{0}+\sum_{i=1}^{l} \frac{p_{i}^{\prime}}{p_{i}}, \quad \text { and } \quad \epsilon=\left(2-l+\sum_{i=1}^{l} \frac{1}{p_{i}}\right) \frac{1}{e} .
$$

In conclusion we have the following result.

Theorem 2.3. ([8]) For any Seifert homology sphere $Y:=\Sigma\left(p_{1}, p_{2}, \ldots, p_{l}\right)$, the Heegaard-Floer homology group $\mathrm{HF}^{+}(-Y)$ is isomorphic to $\mathbb{H}\left(R_{\tau}, \chi_{\tau}\right)$ with a degree shift $-\left(K^{2}+s\right) / 4$, where $\tau$ is the sequence defined in (1.2), and $\left(R_{\tau}, \chi_{\tau}\right)$ is the associated graded root.

Example 2.4. Combining Theorem 2.3 and our Theorem 1.3, it is now easy to calculate the Heegaard-Floer homology of a Seifert homology sphere. Let us illustrate this statement on $Y=$ $\Sigma(2,3,11)$.

We have $N_{0}=5$. Consider $G=G(6,22,33)$, the numerical semigroup generated by the integers 6,22 , and 33 . The only element of $G$ that is contained in the interval $\left[0, N_{0}\right]$ is 0 . Therefore Theorem 1.3 implies $\Delta(0)=1, \Delta(5)=-1$, and $\Delta(n) \geq 0$ for all $n \neq 0,5$. Hence $\tau(n)=\sum_{i=0}^{n-1} \Delta(i)$ has two local minimum values (both of which are equal to 0 ) and one local maximum value (which is equal to 1$)$. Let $\left(R_{\tau}, \chi_{\tau}\right)$ denote the graded root associated with the sequence $\tau=[0,1,0]$. Then $\mathbb{H}\left(R_{\tau}, \chi_{\tau}\right)=\mathcal{T}_{(0)}^{+} \oplus \mathbb{Z}_{(0)}$.

We need to calculate the degree shift $-\left(K^{2}+s\right) / 4$. It follows from (1.1) that the Seifert invariants of $\Sigma(2,3,11)$ are given by

$$
\left(e_{0},\left(p^{\prime}, p\right),\left(q^{\prime}, q\right),\left(r^{\prime}, r\right)\right)=(-2,(1,2),(2,3),(9,11)) .
$$

We calculate the terms appearing in (2.2), and see that $e=-1 / 66, \epsilon=5$. The Dedekind sums are calculated by repeatedly applying the reciprocity law:

$$
\begin{aligned}
s(1,2) & =0, \\
s(2,3) & =-1 / 18, \\
s(9,11) & =-5 / 22 .
\end{aligned}
$$

Using these values in (2.2), the degree shift is calculated to be $-\left(K^{2}+s\right) / 4=-2$. Theorem 1.3 says that $H F^{+}(-\Sigma(2,3,11))=\mathcal{T}_{(-2)}^{+} \oplus \mathbb{Z}_{(-2)}$. 


\section{Analysis of the Delta Function}

In order to determine the positions and values of the local extrema of $\tau$ function, we study its difference term

$$
\Delta(n)=1+e_{0} n-\left\lceil\frac{n p^{\prime}}{p}\right\rceil-\left\lceil\frac{n q^{\prime}}{q}\right\rceil-\left\lceil\frac{n r^{\prime}}{r}\right\rceil .
$$

Our first task is to write $\Delta$ as a quasi-polynomial. To this end, we consider $f: \mathbb{Q} \rightarrow[0,1]$ defined by

$$
f(x):=\lceil x\rceil-x .
$$

Lemma 3.2. Given relatively prime integers $a$ and $m$, the sequence

$$
g(n)=f(n a / m)=\lceil n a / m\rceil-n a / m
$$

is periodic with period $m$. Moreover, the finite sequence $(m g(0), m g(1), \ldots, m g(m-1))$ is the same as the orbit of $m-a$ in the additive group $\mathbb{Z} / m \mathbb{Z}$. Consequently, for every $s \in\{0, \ldots m-1\}$ there exists unique $n$ such that $0 \leq n \leq m-1$ and $f(n a / m)=s / m$.

Proof. Writing $n=p m+r$ we see that $g(n)=g(r)$, establishing the periodicity of $g$. For the second part it suffices to observe that $g(n)$ is the fractional part of $n(m-a) / m$. Indeed,

$$
\frac{n(m-a)}{m}-\left\lfloor\frac{n(m-a)}{m}\right\rfloor=-\frac{n a}{m}-\left\lfloor-\frac{a n}{m}\right\rfloor=-\frac{n a}{m}+\left\lceil\frac{a n}{m}\right\rceil .
$$

We rewrite $\Delta$ accordingly, as follows:

$$
\begin{aligned}
\Delta(n) & =1-e_{0} n-\frac{n p^{\prime}}{p}-\frac{n q^{\prime}}{q}-\frac{n r^{\prime}}{r}-\left(\left\lceil\frac{n p^{\prime}}{p}\right\rceil-\frac{n p^{\prime}}{p}+\left\lceil\frac{n q^{\prime}}{q}\right\rceil-\frac{n q^{\prime}}{q}+\left\lceil\frac{n r^{\prime}}{r}\right\rceil-\frac{n r^{\prime}}{r}\right) \\
& =1-\frac{e_{0} p q r+p^{\prime} q r+p q^{\prime} r+p q r^{\prime}}{p q r} n-\left(f\left(\frac{n p^{\prime}}{p}\right)+f\left(\frac{n q^{\prime}}{q}\right)+f\left(\frac{n r^{\prime}}{r}\right)\right) \\
& =1+\frac{1}{p q r} n-\left(f\left(\frac{n p^{\prime}}{p}\right)+f\left(\frac{n q^{\prime}}{q}\right)+f\left(\frac{n r^{\prime}}{r}\right)\right) \quad \text { (by using (1.1)). }
\end{aligned}
$$

Remark 3.4. The periodic nature of $\Delta$ is now apparent from (3.3). This is suggested by the generating function calculation in Section 7, also. In fact, it follows from generating function calculations that $\Delta$ is the sum of a linear polynomial and a periodic function (which, in turn, can be written as the sum of three periodic functions).

Equality (3.3) allows us to do the following critical analysis regarding the values of $\Delta$. 
Proposition 3.5. For $0 \leq n<$ pqr, we have $-1 \leq \Delta(n) \leq 1$. Moreover,

1. $\Delta(n)=-1$ if and only if $f\left(\frac{n p^{\prime}}{p}\right)+f\left(\frac{n q^{\prime}}{q}\right)+f\left(\frac{n r^{\prime}}{r}\right) \geq 2$.

2. $\Delta(n)=1$ if and only if $f\left(\frac{n p^{\prime}}{p}\right)+f\left(\frac{n q^{\prime}}{q}\right)+f\left(\frac{n r^{\prime}}{r}\right) \leq 1$.

For $n \geq p q r$ we have $\Delta(n) \geq 0$.

Proof. Clearly, $\Delta(0)=1$. Suppose $0<n<p q r$. Let $A(n)=f\left(\frac{n p^{\prime}}{p}\right)+f\left(\frac{n q^{\prime}}{q}\right)+f\left(\frac{n r^{\prime}}{r}\right)$. We have $0<A(n)<3$, and $0<n / p q r<1$. Therefore $\Delta(n)=1+n / p q r-A(n)$ satisfies

$$
-2<\Delta(n)<2
$$

Note that $\Delta(n)$ is integer valued, hence $-1 \leq \Delta(n) \leq 1$ for all $n<p q r$. If $A(n) \geq 2$ then $\Delta(n)<0$, so item 1 follows again from the fact that $\Delta$ is integer valued. Similarly, if $A(n) \leq 1$ then $\Delta>0$, so we obtain the second item.

For $n>p q r$, we have $n / p q r>1$, so $\Delta(n)>-1$ since $A(n)<3$.

Proof of Theorem 1.11. It follows from Proposition 3.5 that the number of times $\Delta$ attains -1 is the number of triples $(x, y, z) \in \mathbb{N}^{3}$ satisfying $0 \leq x \leq p-1,0 \leq y \leq q-1,0 \leq z \leq r-1$ and

$$
\frac{x}{p}+\frac{y}{q}+\frac{z}{r} \geq 2
$$

We interpret this number as the number of lattice points in a tetrahedron as follows. Inequality (3.6) is equivalent to $z \geq 2 r-\frac{r x}{p}-\frac{r y}{q}$. Therefore, we are seeking for the number of lattice points inside the prism $[0, p-1] \times[0, q-1] \times[0, r-1]$ that lie above the hyperplane $\Gamma: z=2 r-\frac{r x}{p}-\frac{r y}{q}$. A straightforward calculation shows that the hyperplane $\Gamma$ intersects $x=p-1$ plane along the line $q z+r y=q r+q r / p$. Similarly, it intersects $y=q-1$ plane along the line $p z+r x=p r+p r / q$. On $z=r-1$ plane we have the line $x+y=p q(r+1) / r$. We depict a generic picture in Figure 2.

It remains to compute the lattice points in the tetrahedron with the vertices $A^{\prime \prime}=(p-1, q-$ $1, r-1), B^{\prime \prime}=(p-1, q-1, r / p+r / q), C^{\prime \prime}=(p-1 q / r+q / p, r-1)$ and $D^{\prime \prime}=(p / r+p / q, q-1, r-1)$. 


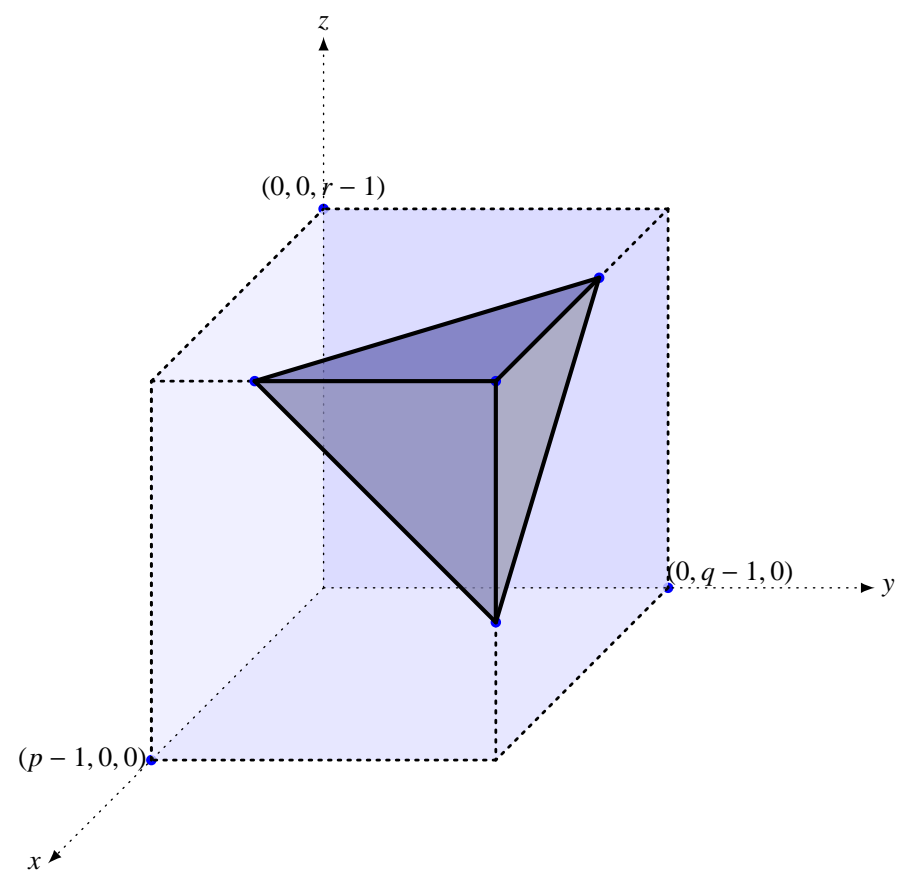

Figure 2: Tetrahedron corresponding to $\Delta=-1$

Shifting the tetrahedron to the origin and simplifying its coordinates give:

$$
\begin{aligned}
A^{\prime} & =(0,0,0) \\
B^{\prime} & =\left(0,0, \frac{p q+q r+p r-p q r}{p q}\right) \\
C^{\prime} & =\left(0, \frac{p q+q r+p r-p q r}{p r}, 0\right) \\
D^{\prime} & =\left(\frac{p q+q r+p r-p q r}{q r}, 0,0\right)
\end{aligned}
$$

The affine transformation $x \mapsto-x, y \mapsto-y$ and $z \mapsto-z$ does not alter the number of points in the tetrahedron:

$$
\begin{aligned}
& A=(0,0,0) \\
& B=\left(0,0,-\frac{p q+q r+p r-p q r}{p q}\right) \\
& C=\left(0,-\frac{p q+q r+p r-p q r}{p r}, 0\right) \\
& D=\left(-\frac{p q+q r+p r-p q r}{q r}, 0,0\right)
\end{aligned}
$$

This proves our claim. 
Lemma 3.7. For positive pairwise relatively prime integers $(p, q, r)$ with $p<q<r$, define $N_{0}(p, q, r)=p q r-p q-q r-p r$. Then $N_{0}\left(p_{1}, q_{1}, r_{1}\right) \leq N_{0}\left(p_{2}, q_{2}, r_{2}\right)$, if $p_{1} \leq p_{2}, q_{1} \leq q_{2}$, and $r_{1} \leq r_{2}$. Consequently $N_{0}(p, q, r)>0$ unless $(p, q, r)=(2,3,5)$.

Proof. Let $P$ denote the set of triples of positive, pairwise relatively prime integers $(p, q, r)$ with $p<q<r$. Consider the partial order on $P$ defined by

$$
\left(p_{1}, q_{1}, r_{1}\right) \leq\left(p_{2}, q_{2}, r_{2}\right) \text { if } p_{1} \leq p_{2}, q_{1} \leq q_{2}, \text { and } r_{1} \leq r_{2}
$$

The triple $(2,3,5)$ is the smallest element of $(P, \leq)$. Define $h(x, y, z)=x y z-x y-y z-x z$ for $x \geq 2, y \geq 3, z \geq 5$, and $x \leq y \leq z$. Then one has $\partial h / \partial x>0, \partial h / \partial y>0$, and $\partial h / \partial z>0$. Therefore $N_{0}$ respects the partial order $\leq$ on $P$. This proves the first assertion. For the second, observe that if $(p, q, r) \in P$ and $(p, q, r) \neq(2,3,5)$, then $(p, q, r) \geq(2,3,7)$, or $(p, q, r) \geq(3,4,5)$. Since $N_{0}(2,3,7)>0$ and $N_{0}(3,4,5)>0$, we have $N_{0}(p, q, r)>0$.

Lemma 3.9. Suppose $(p, q, r) \neq(2,3,5)$. Let $N_{0}=p q r-p q-p r-q r>0$. Then $\Delta\left(N_{0}\right)=-1$, and for all $n>N_{0}$, we have $\Delta(n) \geq 0$.

Proof. The following congruences are easily verified:

$$
\begin{aligned}
& p^{\prime} N_{0} \equiv 1 \bmod p \\
& q^{\prime} N_{0} \equiv 1 \bmod q \\
& r^{\prime} N_{0} \equiv 1 \bmod r
\end{aligned}
$$

Then Lemma 3.2 implies

$$
\begin{aligned}
f\left(\frac{N_{0} p^{\prime}}{p}\right) & =\frac{p-1}{p} \\
f\left(\frac{N_{0} q^{\prime}}{q}\right) & =\frac{q-1}{q} \\
f\left(\frac{N_{0} r^{\prime}}{r}\right) & =\frac{r-1}{r} .
\end{aligned}
$$

Substituting these values in equation 3.3 we get

$$
\Delta\left(N_{0}\right)=2-\left(\frac{1}{p}+\frac{1}{q}+\frac{1}{r}\right)-\left(\frac{p-1}{p}+\frac{q-1}{q}+\frac{r-1}{r}\right)=-1 .
$$


For the second part, using Lemma 3.2 once again, we obtain the following estimate:

$$
f\left(\frac{n p^{\prime}}{p}\right)+f\left(\frac{n q^{\prime}}{q}\right)+f\left(\frac{n r^{\prime}}{r}\right) \leq \frac{p-1}{p}+\frac{q-1}{q}+\frac{r-1}{r}
$$

for all $n$. Suppose $n=N_{0}+s$, for some $s>0$. Then

$$
\Delta(n) \geq 2-\left(\frac{1}{p}+\frac{1}{q}+\frac{1}{r}\right)+\frac{s}{p q r}-\left(\frac{p-1}{p}+\frac{q-1}{q}+\frac{r-1}{r}\right)>-1 .
$$

Hence, $\Delta(n) \geq 0$ for all $n>N_{0}$.

Next, we prove that $\Delta$-function is centrally symmetric with respect to $N_{0} / 2$.

Lemma 3.10. Let $N_{0}=p q r-p q-q r-p r$. For any integer $i$ such that $0 \leq i \leq N_{0}$, we have

$$
\Delta(i)=-\Delta\left(N_{0}-i\right)
$$

Proof. Let $a, b$, and $c$ be three integers defined by the conditions

$$
\begin{aligned}
& a \equiv i p^{\prime} \bmod p, \quad 0 \leq a \leq p-1, \\
& b \equiv i q^{\prime} \bmod q, \quad 0 \leq a \leq q-1, \\
& c \equiv i r^{\prime} \bmod r, \quad 0 \leq a \leq r-1 .
\end{aligned}
$$

Then by (3.3) we have

$$
\begin{aligned}
\Delta(i) & =1+\frac{i}{p q r}-\left(f\left(\frac{a}{p}\right)+f\left(\frac{b}{q}\right)+f\left(\frac{c}{r}\right)\right) \\
\Delta\left(N_{0}-i\right) & =2-\frac{1}{p}-\frac{1}{q}-\frac{1}{r}-\frac{i}{p q r}-\left(f\left(\frac{1-a}{p}\right)+f\left(\frac{1-b}{q}\right)+f\left(\frac{1-c}{r}\right)\right) .
\end{aligned}
$$

After adding these two equations and plugging the definition of $f$ in, and doing the obvious cancellations, we see that

$$
\Delta(i)+\Delta\left(N_{0}-i\right)=3-\left(\left\lceil\frac{1-a}{p}\right\rceil+\left\lceil\frac{a}{p}\right\rceil+\left\lceil\frac{1-b}{q}\right\rceil+\left\lceil\frac{b}{q}\right\rceil+\left\lceil\frac{1-c}{r}\right\rceil+\left\lceil\frac{c}{r}\right\rceil\right) .
$$

Hence the following lemma finishes the proof

Lemma 3.11. Let $a$ and $p$ be integers such that $0 \leq a \leq p-1$. Then

$$
\left\lceil\frac{1-a}{p}\right\rceil+\left\lceil\frac{a}{p}\right\rceil=1
$$


Proof. If $a \neq 0$, then the first term is 0 and the other one is 1 . If $a=0$ then the first term is 1 and the other one is 0 . In both cases they add up to 1 .

Proof of Theorem 1.3. The first four items follow from Lemma 3.7, Lemma 3.9, Lemma 3.10, and Proposition 3.5, respectively. The proof of the second part of Lemma 3.9 shows that $\Delta(n) \geq 0$ when $(p, q, r)=(2,3,5)$. It remains proving the fifth item.

Let $G=G(p q, p r, q r)$ denote the semigroup generated by $0, p q$, $p r$, and $q r$. If $n \in G$, then $n=a q r+b p r+c p q$ for some $a, b, c \geq 0$. Hence, we see that

$$
\begin{aligned}
n p^{\prime} & \equiv-a \bmod p, \\
n q^{\prime} & \equiv-b \bmod q, \\
n r^{\prime} & \equiv-c \bmod r .
\end{aligned}
$$

Let $\widetilde{a}, \widetilde{b}$ and $\widetilde{c}$ denote the residues of $a, b, c$ modulo $p, q, r$, respectively. Then Lemma 3.2 implies

$$
f\left(\frac{n p^{\prime}}{p}\right)+f\left(\frac{n q^{\prime}}{q}\right)+f\left(\frac{n r^{\prime}}{r}\right)=\frac{\widetilde{a}}{p}+\frac{\widetilde{b}}{q}+\frac{\widetilde{c}}{r} .
$$

Plugging in (3.3), we get $\Delta(n) \geq 1$. It follows from Proposition 3.5 that $\Delta(n)=1$, if $n \in G \cap\left[0, N_{0}\right]$.

Next, we show that $G \cap\left[0, N_{0}\right]$ contains all the elements $n \in\left[0, N_{0}\right]$ with $\Delta(n)=1$. We prove this by showing that the cardinalities of these two sets are equal. Indeed, by the symmetry proven in Lemma 3.10 the number of times $\Delta$ attains +1 in $\left[0, N_{0}\right]$ is equal to the number of times $\Delta$ attains -1 in the same interval. On the other hand, we know from Theorem 1.11 that the total number of -1 's of $\Delta$ is equal to the cardinality of $G \cap\left[0, N_{0}\right]$. Therefore, the proof is complete.

Next we establish the monotonicity of $\kappa$ on the set of ordered triples with respect to the natural partial order $\leq$ defined in (3.8).

Proposition 3.12. Suppose $\left(p_{1}, q_{1}, r_{1}\right) \geq\left(p_{2}, q_{2}, r_{2}\right)$, then $\kappa\left(p_{1}, q_{1}, r_{1}\right) \geq \kappa\left(p_{2}, q_{2}, r_{2}\right)$.

Proof. In view of Theorem 1.11, it suffices to show that the tetrahedron $T_{1}$ corresponding to $\left(p_{1}, q_{1}, r_{1}\right)$ contains the tetrahedron $T_{2}$ corresponding to $\left(p_{2}, q_{2}, r_{2}\right)$. The edge of $T_{1}$ on the $x$-axis has length

$$
l_{x}\left(T_{1}\right)=r_{1}\left(1-\frac{1}{p_{1}}-\frac{1}{q_{1}}\right)-1
$$


so, the hypothesis implies that $l_{x}\left(T_{1}\right) \geq l_{x}\left(T_{2}\right)$. By symmetry, the edges on the $y$-, and the $z$-axes satisfy the same property, hence, the proof follows.

\section{Generalizations}

In this section we extend our results to Seifert homology spheres with four or more singular fibers. We start with a modified version of Theorem 1.3.

Theorem 4.1. For $l \geq 4$, let $\left(p_{1}, p_{2}, \ldots, p_{l}\right)$ be an l-tuple of pairwise relatively prime integers with $1<p_{1}<p_{2}<\cdots<p_{l}$. Let $\Delta: \mathbb{N} \rightarrow \mathbb{Z}$ denote the function

$$
\Delta(n)=1+\left|e_{0}\right| n-\sum_{i=1}^{l}\left\lceil\frac{n p_{i}^{\prime}}{p_{i}}\right\rceil,
$$

where $\left(e_{0}, p_{1}^{\prime}, p_{2}^{\prime}, \ldots, p_{l}^{\prime}\right)$ is defined by the equation

$$
e_{0} p_{1} p_{2} \cdots p_{l}+p_{1}^{\prime} p_{2} \cdots p_{l}+p_{1} p_{2}^{\prime} \cdots p_{l}+\cdots+p_{1} p_{2} \cdots p_{l}^{\prime}=-1,
$$

with $0 \leq p_{i}^{\prime} \leq p_{i}-1$, for all $i=1, \ldots, l$. Define the constant

$$
N_{0}=p_{1} p_{2} \cdots p_{l}\left((l-2)-\sum_{i=1}^{l} \frac{1}{p_{i}}\right) \in \mathbb{Z}_{>0}
$$

Then

1. $\Delta(n) \geq 0$, for all $n>N_{0}$.

2. $\Delta(n)=-\Delta\left(N_{0}-n\right)$, for $0 \leq n \leq N_{0}$.

3. $\Delta(n) \in\{-(l-2), \ldots,-1,0,1, \ldots, l-2\}$, for $0 \leq n \leq N_{0}$.

4. For $0 \leq n \leq N_{0}$, one has $\Delta(n) \geq 1$ if and only if either $n=0$, or $n$ is an element of the numerical semigroup $G$ minimally generated by $p_{1} p_{2} \ldots p_{l} / p_{i}$ for $i=1,2, \ldots, l$.

5. If $n \in G$ is of the form $n=p_{1} p_{2} \ldots p_{l} \sum_{i=1}^{l} \frac{x_{i}}{p_{i}}$, then $\Delta(n)=1+\sum_{i=1}^{l}\left\lfloor\frac{x_{i}}{p_{i}}\right\rfloor$.

We omit the proofs of items 1-3, since they are identical to the case $l=3$, except that one needs a generalization of Equation 3.3 to write $\Delta(n)$ as a linear quasi-polynomial.

$$
\Delta(n)=1+\frac{n}{p_{1} \ldots p_{l}}-\sum_{i=1}^{l} f\left(\frac{n p_{i}^{\prime}}{p_{i}}\right)
$$


The proofs of items 4 and 5 are postponed to the end of the chapter. The proof of item 4 relies on a generalization of Theorem 1.11 which we discuss now.

Let $\left(p_{1}, p_{2}, \ldots, p_{l}\right)$ be an $l$-tuple of pairwise relatively prime integers with $1<p_{1}<\cdots<p_{l}$. In the case where $l=3$, it is readily known that $\kappa\left(p_{1}, p_{2}, p_{3}\right)$ equals the number of lattice points in a tetrahedron. For $l \geq 4, \kappa\left(p_{1}, p_{2}, \ldots, p_{l}\right)$ is still equal to the number of lattice points in a polytope, however, the polytope is not necessarily a tetrahedron. Another difference is that, this time each lattice point is counted with a certain multiplicity. To state our result we need more notation. Define

$$
N_{0}(k)=p_{1} \ldots p_{l}\left(k-\sum_{i=1}^{l} \frac{1}{p_{i}}\right) .
$$

Let $H_{k}$ be the hyperplane in $\mathbb{R}^{l}$ defined by

$$
\sum_{i=1}^{l} \frac{x_{i}}{p_{i}}=\frac{N_{0}(k)}{p_{1} p_{2} \ldots p_{l}} .
$$

Suppose $k \in\{1,2, \ldots, l-2\}$ with $N_{0}(k)>0$. Then $H_{k}$ cuts out a tetrahedron $T_{k}$ from the first orthant of $\mathbb{R}^{l}$. For convenience, we define $T_{k}=\emptyset$ if $N_{0}(k)<0$. Then we have

$$
T_{l-2} \supset T_{l-3} \supset \cdots T_{1} \supset T_{0}
$$

Let $C=C_{p_{1}, \ldots, p_{l}}$ denote the $l$-dimensional cube $\left[0, p_{1}-1\right] \times\left[0, p_{2}-1\right] \cdots \times\left[0, p_{l}-1\right] \subset \mathbb{R}^{l}$, and set

$$
A_{k}=\#\left(\left(T_{k}-T_{k-1}\right) \cap C \cap \mathbb{Z}^{l}\right)
$$

In other words, $A_{k}$ is the number of lattice points from $C$ that lie between the hyperplanes $H_{k}$ and $H_{k-1}$. Note that $A_{l-2}>0$, and $A_{j}=0$ if $N_{0}(j)<0$.

Recall that the $\kappa$-invariant is equal to the sum of all negative values of $\Delta(n)$.

\section{Theorem 4.5.}

$$
\kappa\left(p_{1}, p_{2}, \ldots, p_{l}\right)=\sum_{j=1}^{l-2} \frac{(l-j-1)(l-j)}{2} A_{j}
$$

Proof. We begin with describing a useful affine transformation on $\mathbb{R}^{l}$. Let $\varphi: \mathbb{R}^{l} \rightarrow \mathbb{R}^{l}$ denote the map defined by $\varphi\left(x_{1}, x_{2}, \ldots, x_{l}\right)=\left(y_{1}, y_{2}, \ldots, y_{l}\right)$, where $y_{i}=-\left(x_{i}-\left(p_{i}-1\right)\right)$ for $i=1,2, \ldots, l$. Clearly, $C$ is invariant under $\varphi$, and moreover, $\varphi$ maps the hyperplane $\widetilde{H}_{k}$ defined by

$$
\sum_{i=1}^{l} \frac{x_{i}}{p_{i}}=l-k
$$


to the hyperplane $H_{k}$ defined in (4.3). Let $\widetilde{A_{k}}$ denote the number of lattice points in $C$ that lie between $\widetilde{H}_{k-1}$ and $\widetilde{H}_{k}$. Then $\widetilde{A}_{k}=A_{k}$ for every $k \in\{1,2, \ldots, l-2\}$.

Next, we compute the sum of negative values of $\Delta$. By Lemma 3.2, each $f\left(\frac{n p_{i}^{\prime}}{p_{i}}\right)$ term equals $\frac{x_{i}}{p_{i}}$ for some $x_{i} \in\left\{0,1, \ldots, p_{i}-1\right\}$. Hence, (4.2) implies that $\Delta(n) \geq 0$ for $n \geq(l-2) p_{1} \ldots p_{l}$. Therefore, it remains to find the sum of negative values of $\Delta(n)$ for $(k-1) p_{1} \ldots p_{l} \leq n<k p_{1} \ldots p_{l}$, where $k \in\{0,1, \ldots, l-3\}$,

Notice that, in the interval $(k-1) p_{1} \ldots p_{l} \leq n<k p_{1} \ldots p_{l}$, we have $\Delta(n) \geq-(l-k-1)$. Let $t \in\{1,2, \ldots, l-k-1\}$ and let $B(k, t)$ denote the number of times $\Delta$ attains the value $-t$ in the interval $(k-1) p_{1} \ldots p_{l} \leq n<k p_{1} \ldots p_{l}$. It follows from (4.2) that $B(k, t)$ is equal to the number of integers $n$ which solve the inequality

$$
t+k+1 \geq \sum_{i=1}^{l} f\left(\frac{n p_{i}^{\prime}}{p_{i}}\right) \geq t+k,
$$

with $(k-1) p_{1} \ldots p_{l} \leq n<k p_{1} \ldots p_{l}$.

Observe that the Chinese remainder theorem combined with Lemma 3.2 implies that given any $l$-tuple $\left(x_{1}, \ldots, x_{l}\right)$ with $x_{i} \in\left\{0,1, \ldots, p_{i}-1\right\}$, there exists unique $n$ in the interval $(k-1) p_{1} \ldots p_{l} \leq$ $n<k p_{1} \ldots p_{l}$ such that $f\left(\frac{n p_{i}^{\prime}}{p_{i}}\right)=\frac{x_{i}}{p_{i}}$ for $i \in\{1, \ldots, l\}$. Thus, in the light of inequality (4.6), we see that $B(k, t)$ is equal to the number of lattice points in $C$ that lie between $\widetilde{H}_{l-t-k}$ and $\widetilde{H}_{l-t-k-1}$. In other words $B(k, t)=\widetilde{A_{l-t-k}}$. Finally, by the following manipulations we finish the proof.

$$
\begin{aligned}
\kappa\left(p_{1}, p_{2}, \ldots, p_{l}\right) & =\left|\sum_{n=0}^{\infty} \min \{0, \Delta(n)\}\right|=\left|\sum_{k=1}^{l-2} \sum_{n=(k-1) p_{1} \ldots p_{l}}^{k p_{1} \ldots p_{l}} \min \{0, \Delta(n)\}\right| \\
& =\sum_{k=1}^{l-2} \sum_{t=1}^{l-k-1} t B(k, t)=\sum_{k=1}^{l-2} \sum_{t=1}^{l-k-1} t \widetilde{A_{l-t-k}} \\
& =\sum_{k=1}^{l-2} \sum_{t=1}^{l-k-1} t A_{l-t-k}=\sum_{k=1}^{l-2} \sum_{j=1}^{l-k-1}(l-k-j) A_{j} \\
& =\sum_{j=1}^{l-2} \sum_{k=1}^{l-j-1}(l-k-j) A_{j} \\
& =\sum_{j=1}^{l-2} \frac{(l-j-1)(l-j)}{2} A_{j} .
\end{aligned}
$$


In order for proving part 4 of Theorem 4.1, we need to relate the count of lattice points given in Theorem 4.5 to the number of lattice points in the tetrahedra $T_{k}$. This relation is established with the help of the function $\pi: \mathbb{Z}^{l} \rightarrow C \cap \mathbb{Z}^{l}$ defined by $\pi\left(x_{1}, x_{2}, \ldots, x_{l}\right)=\left(z_{1}, z_{2}, \ldots, z_{l}\right)$, where $z_{i} \equiv x_{i}\left(\bmod p_{i}\right)$ with $0 \leq z_{i} \leq p_{i}-1$ for $i=1, \ldots, l$.

Lemma 4.7. Suppose $k \in\{1,2, \ldots, l-2\}$ with $N_{0}(k)>0$. Then

$$
\pi\left(\left(T_{k} \backslash T_{k-1}\right) \cap \mathbb{Z}^{l}\right)=T_{k} \cap C \cap \mathbb{Z}^{l}
$$

Proof. The inclusion

$$
\pi\left(\left(T_{k} \backslash T_{k-1}\right) \cap \mathbb{Z}^{l}\right) \subset T_{k} \cap C \cap \mathbb{Z}^{l}
$$

is obvious. To prove the reverse inclusion let $\left(z_{1}, z_{2}, \ldots, z_{l}\right)$ be a point from $T_{k} \cap C \cap \mathbb{Z}^{l}$, and let $r$ be the unique non-negative integer satisfying

$$
\frac{N_{0}(k)}{p_{1} p_{2} \ldots p_{l}} \geq r+\sum_{i=1}^{l} \frac{z_{i}}{p_{i}}>\frac{N_{0}(k-1)}{p_{1} p_{2} \ldots p_{l}} .
$$

Let $r_{1}, \ldots, r_{l}$ be non-negative numbers such that $r=r_{1}+\cdots+r_{l}$. Define $x_{i}=z_{i}+r_{i} p_{i}, i=1, \ldots, l$. Then $\pi\left(x_{1}, x_{2}, \ldots, x_{l}\right)=\left(z_{1}, z_{2}, \ldots, z_{l}\right)$. It follows from (4.8) that

$$
\frac{N_{0}(k)}{p_{1} p_{2} \ldots p_{l}} \geq \sum_{i=1}^{l} \frac{x_{i}}{p_{i}}>\frac{N_{0}(k-1)}{p_{1} p_{2} \ldots p_{l}} .
$$

Hence $\left(x_{1}, x_{2}, \ldots, x_{l}\right) \in T_{k} \backslash T_{k-1}$ as required.

Lemma 4.9. Let $k \geq k^{\prime}$ be two elements from $\{1,2, \ldots, l-2\}$ with $N_{0}(k) \geq N_{0}\left(k^{\prime}\right)>0$. Let $\left(x_{1}, x_{2}, \ldots, x_{l}\right) \in\left(T_{k} \backslash T_{k-1}\right) \cap \mathbb{Z}^{l}$. Then $\pi\left(x_{1}, x_{2}, \ldots, x_{l}\right) \in\left(T_{k^{\prime}} \backslash T_{k^{\prime}-1}\right) \cap C \cap \mathbb{Z}^{l}$ if and only if

$$
\sum_{i=1}^{l}\left\lfloor\frac{x_{i}}{p_{i}}\right\rfloor=k-k^{\prime}
$$

Proof. Let $\left(z_{1}, z_{2}, \ldots, z_{l}\right)=\pi\left(x_{1}, x_{2}, \ldots, x_{l}\right)$. Then there exist non-negative integers $r_{1}, r_{2}, \ldots, r_{l}$ such that $x_{i}=z_{i}+r_{i} p_{i}, i=1,2, \ldots, l$. Hence,

$$
\sum_{i=1}^{l}\left\lfloor\frac{x_{i}}{p_{i}}\right\rfloor=\sum_{i=1} r_{i}
$$

Then as in the proof of Lemma 4.7, we have that

$$
\frac{N_{0}(k)}{p_{1} p_{2} \ldots p_{l}} \geq \sum_{i=1}^{l} \frac{x_{i}}{p_{i}}>\frac{N_{0}(k-1)}{p_{1} p_{2} \ldots p_{l}} .
$$


Suppose now $\left(z_{1}, z_{2}, \ldots, z_{l}\right) \in\left(T_{k^{\prime}} \backslash T_{k^{\prime}-1}\right) \cap C \cap \mathbb{Z}^{l}$. Then

$$
\frac{N_{0}\left(k^{\prime}\right)}{p_{1} p_{2} \ldots p_{l}} \geq \sum_{i=1}^{l} \frac{z_{i}}{p_{i}}>\frac{N_{0}\left(k^{\prime}-1\right)}{p_{1} p_{2} \ldots p_{l}}
$$

Combining (4.11) with (4.12), we see that

$$
k-k^{\prime}-1<\sum_{i=1}^{l} \frac{x_{i}-z_{i}}{p_{i}}<k-k^{\prime}+1
$$

Note that the middle term in the above inequality is an integer, and therefore,

$$
\sum_{i=1}^{l} r_{i}=k-k^{\prime}
$$

The desired equality now follows from (4.10).

$$
\begin{aligned}
& \text { Conversely, assume that } \sum_{i=1}^{l} r_{i}=k-k^{\prime} \text {. By (4.11) } \\
& \qquad \frac{N_{0}(k)}{p_{1} p_{2} \ldots p_{l}} \geq k-k^{\prime}+\sum_{i=1}^{l} \frac{z_{i}}{p_{i}}>\frac{N_{0}(k-1)}{p_{1} p_{2} \ldots p_{l-1}} .
\end{aligned}
$$

Hence

$$
\frac{N_{0}\left(k^{\prime}\right)}{p_{1} p_{2} \ldots p_{l}} \geq \sum_{i=1}^{l} \frac{z_{i}}{p_{i}}>\frac{N_{0}\left(k^{\prime}-1\right)}{p_{1} p_{2} \ldots p_{l-1}}
$$

which implies $\left(z_{1}, z_{2}, \ldots, z_{l}\right) \in\left(T_{k^{\prime}} \backslash T_{k^{\prime}-1}\right) \cap C \cap \mathbb{Z}^{l}$.

Proof of Theorem 4.1. Proofs of parts 1-3 are similar to those in Theorem 1.3. Part 5 is a direct consequence of Equation 4.2. Indeed, plugging $n=p_{1} p_{2} \ldots p_{l} \sum_{i=1}^{l} \frac{x_{i}}{p_{i}}$ in (4.2), we see that

$$
\begin{aligned}
\Delta(n) & =1+\sum_{i=1}^{l} \frac{x_{i}}{p_{i}}-\sum_{i=1}^{l} f\left(\frac{-x_{i}}{p_{i}}\right) \\
& =1-\sum_{i=1}^{l}\left\lceil-\frac{x_{i}}{p_{i}}\right\rfloor \quad \text { by (3.1). } \\
& =1+\sum_{i=1}^{l}\left\lfloor\frac{x_{i}}{p_{i}}\right\rfloor \quad
\end{aligned}
$$


What is left is to show that $\Delta(n)\left(n \leq N_{0}(l-2)\right)$ attains all its positive values on $G$. Note that

$$
\kappa\left(p_{1}, p_{2}, \ldots, p_{l}\right)=\left|\sum_{n=0}^{\infty} \min \{0, \Delta(n)\}\right|=\left|\sum_{n=0}^{N_{0}} \min \{0, \Delta(n)\}\right|=\left|\sum_{n=0}^{N_{0}} \max \{0, \Delta(n)\}\right|,
$$

where the second and third equalities are due to parts 2 and 3 respectively. Define

$$
\kappa^{\prime}\left(p_{1}, p_{2}, \ldots, p_{l}\right):=\sum_{n \in G \cap\left[0, N_{0}\right]} \Delta(n)
$$

By part 6, we have

$$
\kappa^{\prime}\left(p_{1}, p_{1}, \ldots, p_{l}\right) \leq \kappa\left(p_{1}, p_{1}, \ldots, p_{l}\right) .
$$

Obviously, this inequality is strict if $\Delta(n)$ attains a positive value outside of the semigroup $G$. We prove that this is not the case.

Using its minimal generating set, we represent the elements of $G$ by $l$-tuples as follows. Let $\phi: \mathbb{Z}^{l} \rightarrow G$ denote the map defined by

$$
\phi\left(x_{1}, \ldots, x_{l}\right)=p_{1} p_{2} \ldots p_{l} \sum_{i=1}^{l} \frac{x_{i}}{p_{i}}
$$

Clearly, $\phi$ is a finite-to-one map. Let $R\left(x_{1}, x_{2}, \ldots, x_{l}\right)$ denote the cardinality of the set $\phi^{-1}\left(\phi\left(x_{1}, x_{2}, \ldots, x_{l}\right)\right)$. We are interested in the role that $R\left(x_{1}, x_{2}, \ldots, x_{l}\right)$ plays in the computation of $\kappa^{\prime}$, rather than its actual value.

$$
\begin{aligned}
\kappa^{\prime}\left(p_{1}, p_{2}, \ldots, p_{l}\right) & =\sum_{\substack{\left(x_{1}, x_{2}, \ldots, x_{l}\right) \in T_{l-2} \cap \mathbb{Z}^{l}\\
}} \frac{\Delta\left(\phi\left(x_{1}, x_{2}, \ldots, x_{l}\right)\right)}{R\left(x_{1}, x_{2}, \ldots, x_{l}\right)} \\
& \sum_{\substack{k=1 \\
N_{0}(k)>0}}^{l-2} \sum_{\substack{\left(x_{1}, x_{2}, \ldots, x_{l}\right) \in\left(T_{k} \backslash T_{k-1}\right) \cap \mathbb{Z}^{l} \\
R\left(x_{1}, x_{2}, \ldots, x_{l}\right)}} \frac{1}{l}\left\lfloor\sum_{i=1}^{l}\left\lfloor\frac{x_{i}}{p_{i}}\right\rfloor\right) .
\end{aligned}
$$

Here, the second equality is a consequence of part 5. We need to count the elements appearing in the second sum.

We know from Lemma 4.7 that $\pi\left(\left(T_{k} \backslash T_{k-1}\right) \cap \mathbb{Z}^{l}\right)=T_{k} \cap C \cap \mathbb{Z}^{l}$, for $k=1, \ldots, l-2$. Moreover, two $l$-tuples $\left(x_{1}, x_{2}, \ldots, x_{l}\right),\left(y_{1}, y_{2}, \ldots, y_{l}\right) \in\left(T_{k} \backslash T_{k-1}\right) \cap \mathbb{Z}^{l}$ are mapped to the same element by $\pi$ if and only if they are mapped onto the same element by $\phi$. These observations combined with 
Lemma 4.9 gives

$$
\begin{aligned}
\kappa^{\prime}\left(p_{1}, p_{2}, \ldots, p_{l}\right) & =\sum_{\substack{k=1 \\
N_{0}(k)>0}}^{l-2} \sum_{\substack{j=1 \\
N_{0}(j)>0}}^{k} \sum_{\left(z_{1}, z_{2}, \ldots, z_{l}\right) \in\left(T_{j} \backslash T_{j-1}\right) \cap C \cap \mathbb{Z}^{l}} k-j+1 \\
& =\sum_{\substack{k=1 \\
N_{0}(k)>0}}^{l-2} \sum_{\substack{j=1 \\
N_{0}(j)>0}}^{k}(k-j+1) A_{j} \quad \text { (by (4.4)). }
\end{aligned}
$$

Note that the above equation is valid even without the restrictions $N_{0}(k) \geq N_{0}(j)>0$, since we force $A_{j}=0$, if $N_{0}(j)<0$. Changing the order of the summation and the indices accordingly give

$$
\begin{aligned}
\kappa^{\prime}\left(p_{1}, p_{2}, \ldots, p_{l}\right) & =\sum_{j=1}^{l-2} \sum_{k=j}^{l-2}(k-j+1) A_{j}=\sum_{j=1}^{l-2} \sum_{r=1}^{l-1-j} r A_{j} \\
& =\sum_{j=1}^{l-2} \frac{(l-j-1)(l-j)}{2} A_{j} .
\end{aligned}
$$

Hence by Theorem 4.5, we have $\kappa^{\prime}\left(p_{1}, p_{2}, \ldots, p_{l}\right)=\kappa\left(p_{1}, p_{2}, \ldots, p_{l}\right)$.

Next we establish the monotonicity of $\kappa$ under the addition of one more singular fiber.

Proposition 4.13. Let $\left(p_{1}, p_{2}, \ldots, p_{l}, p_{l+1}\right)$ be an $(l+1)$-tuple of pairwise relatively prime integers with $1<p_{1}<p_{2}<\cdots<p_{l}<p_{l+1}$. Then

$$
\kappa\left(p_{1}, p_{2}, \ldots, p_{l}\right) \leq \kappa\left(p_{1}, p_{2}, \ldots, p_{l}, p_{l+1}\right)
$$

Proof. Let $\Delta$ and $\Delta^{\prime}$ be the difference terms corresponding to the $l$-tuple $\left(p_{1}, p_{2}, \ldots, p_{l}\right)$ and the $(l+1)$-tuple $\left(p_{1}, p_{2}, \ldots, p_{l}, p_{l+1}\right)$ respectively. Let $n$ be an integer with $\Delta(n) \leq 0$. We claim that $\Delta^{\prime}(n) \leq \Delta(n)$. Writing the difference terms as in (3.3), we have

$$
\Delta^{\prime}(n)-\Delta(n)=\frac{n}{p_{1} p_{2} \ldots p_{l} p_{l+1}}-\frac{n}{p_{1} p_{2} \ldots p_{l}}-f\left(\frac{n p_{l+1}^{\prime}}{p_{l+1}}\right) \leq 0 .
$$

We state the monotonicity of kappa under the natural partial order of $l$-tuples, generalizing Proposition 3.12.

Proposition 4.14. Suppose $\left(p_{1}, p_{2}, \ldots, p_{l}\right) \geq\left(q_{1}, q_{2}, \ldots, q_{l}\right)$, then $\left.\kappa\left(p_{1}, p_{2}, \ldots, p_{l}\right)\right) \geq \kappa\left(q_{1}, q_{2}, \ldots, q_{l}\right)$. 
Proof. From the discussion preceding Theorem 4.5, each tetrahedron associated to $\left(p_{1}, p_{2}, \ldots, p_{l}\right)$ is strictly larger than the corresponding tetrahedron associated to $\left(q_{1}, q_{2}, \ldots, q_{l}\right)$. Hence the monotonicity follows from the count given in Theorem 4.5. Indeed, every lattice point appearing in the calculation of $\kappa\left(p_{1}, p_{2}, \ldots, p_{l}\right)$ appears also in the calculation of $\kappa\left(q_{1}, q_{2}, \ldots, q_{l}\right)$ with a possibly bigger multiplicity. This is because of the fact that the lattice points in the smaller tetrahedra are counted with bigger multiplicity in Theorem 4.5 .

\section{Topological Applications}

In this section we discuss some of the topological applications of our work to the topology of 3manifolds. Our first task is to detect the Brieskorn spheres with trivial Heegaard-Floer homology. We would like to find all Brieskorn spheres which are $L$-spaces, so, we first translate the condition to being an $L$-space in terms of the tau function defined in (1.2).

Proposition 5.1. Let $Y$ be a 3-manifold which bounds a negative definite plumbing with at most one bad vertex. Then $Y$ is an L-space if and only if its tau function is increasing.

Proof. It follows from Nemethi's work that Heegaard-Floer homology in the canonical Spin ${ }^{c}$ structure is given by the graded root associated with its tau function. In particular, this gives trivial homology if and only $\tau$ is increasing. Now, the proof follows from Theorem 6.3 of Nemethi [8], which states that a plumbed 3-manifold is an $L$-space if and only if its Heegaard-Floer homology in the canonical $\operatorname{Spin}^{c}$ structure is trivial.

It is known that the 3-sphere and the Poincare homology sphere $\Sigma(2,3,5)$ are examples of $L$-spaces. In fact it is conjectured that an irreducible integral homology sphere is an $L$-space if and only if it is homeomorphic to $S^{3}$, or to $\Sigma(2,3,5)$ (with either orientation). Here we verify this conjecture for Seifert homology spheres. This was observed long before by Rustamov and independently by Eftekhary, but here we give a simpler proof.

Proof of Theorem 1.6. In view of Proposition 5.1, it suffices to prove the following: If $\tau$ function of a Seifert homology sphere $Y:=\Sigma\left(p_{1}, p_{2}, \ldots, p_{l}\right)$ is increasing, then either $l \leq 2$ (implying $\left.Y \approx S^{3}\right)$, or $l=3$ and $\left(p_{1}, p_{2}, p_{3}\right)=(2,3,5)$. That $\tau$ is increasing is equivalent to the condition that $\Delta(n)=\tau(n+1)-\tau(n) \geq 0$. Then Theorem 4 .1 rules out the possibility that $l \geq 4$, since $\Delta\left(N_{0}\right)=-\Delta(0)=-1$. If $l=3$, Theorem 1.3 forces that $\left(p_{1}, p_{2}, p_{3}\right)=(2,3,5)$. 
Proof of Proposition 1.10. This is an immediate consequence of Theorem 2.3, and the relationship between Heegaard-Floer homology and the Casson invariant. More precisely, Ozsváth and Szabó show in [11] that for every integral homology sphere $Y$, the Heegaard-Floer homology has a decomposition of the form

$$
H F^{+}(-Y)=\mathcal{T}_{(d)}^{+} \oplus H F_{\text {red }}(-Y)
$$

where $H F_{\text {red }}(-Y)$ is a finitely generated subgroup, whose Euler characteristic satisfies the following property:

$$
\chi\left(H F_{\text {red }}(-Y)\right)=\lambda(-Y)+\frac{d(-Y)}{2} .
$$

It is shown in [12] that if $Y$ is Seifert homology sphere (or more generally if $Y$ bounds a negative definite plumbing with at most one bad vertex), then $\mathrm{HF}^{+}(-Y)$ is supported only in even degrees. Hence, $\chi\left(H F_{\text {red }}(-Y)\right)=\operatorname{rank}\left(H F_{\text {red }}(-Y)\right)$ for every Seifert homology sphere $Y$. By the discussion in Section 2, we read off this quantity from the corresponding graded root directly: Simply remove the longest branch, then the number of remaining vertices is the rank of $H F_{\text {red }}(-Y)$. By Theorem 2.3 , the graded root is determined by the tau function.

It is straightforward to verify $\operatorname{rank}\left(\mathbb{H}_{\text {red }}\left(R_{\tau}\right), \chi_{\tau}\right)=\min _{i} \tau(i)+\sum_{i} \max \{-\Delta(i), 0\}$ (see Corollary 3.7 of [8]). Comparing with Definition 1.7, we have $\kappa\left(p_{1}, \ldots, p_{l}\right)=\sum_{i} \max \{-\Delta(i), 0\}$. Substituting in (5.2) we obtain

$$
\kappa=\lambda(-Y)+\frac{d(-Y)}{2}-\min _{i} \tau(i)
$$

The theorem then follows from the fact that $\frac{d(-Y)}{2}-\min _{i} \tau(i)$ is the half of the degree shift term $\left(K^{2}+s\right) / 4$, which is discussed in Section 2.

Proof of Theorem 1.9. Using Theorem 1.3 and Nemethi's method described in Section 2, it is easy to verify Table 1 . We must show that every Seifert homology sphere has $\kappa \geq 3$, except the ones given in Table 1 . Let $\Sigma\left(p_{1}, p_{2}, \ldots, p_{l}\right)$ be a Seifert homology sphere that does not appear in 1. Then $l \geq 3$ since only Seifert homology with less than 3 singular fibers is $S^{3}$. Suppose $l=3$, then the triple $\left(p_{1}, p_{2}, p_{3}\right)$ must be greater than or equal to one of the following triples: $(3,5,7)$, $(3,4,7),(3,5,9),(2,7,9),(2,5,11),(2,5,13),(2,5,19),(2,3,19)$. These triples are the immediate successors of the triples appearing in the table. It is easy to check that all of these triples have $\kappa \geq 3$, so by monotonicity we are done in the case of three singular fibers. For four and more singular fibers, we have $\kappa\left(p_{1}, p_{2}, \ldots, p_{l}\right) \geq \kappa(2,3,5,7) \geq \kappa(3,5,7) \geq 4$. 
We are ready to prove Theorem 1.8 , which states that there are only finitely many Seifert homology spheres with a prescribed $\kappa$, and therefore, a prescribed Heegaard-Floer homology.

Proof of Theorem 1.8. We already know that when $\kappa=0$, there are only two possible Seifert homology spheres, namely, $S^{3}$, or the Poincaré homology sphere. For the general case, it is enough to show that $\kappa$ is not constant on any infinite family of Seifert homology spheres each of which contains three or more singular fibers.

We begin with families of Brieskorn spheres. Let $\left\{\left(p_{n}, q_{n}, r_{n}\right): n=1, \ldots, \infty\right\}$ be an infinite family of triples. Since $p_{n}<q_{n}<r_{n}$, the last entry $r_{n}$ can not stay constant. Hence, after passing to a subsequence we may assume that $\left(p_{n}, q_{n}, r_{n}\right)$ is increasing with $r_{n} \rightarrow \infty$. This implies that $\kappa\left(p_{n}, q_{n}, r_{n}\right) \rightarrow \infty$ by Proposition 3.12 and its proof. In particular $\kappa\left(p_{n}, q_{n}, r_{n}\right)$ is not constant. Suppose now that we have infinite family of Seifert homology spheres $\left(p_{1, n}, p_{2, n}, \ldots, p_{l(n), n}\right)$ with $l(n) \geq 3$ for all $n$. Projecting to the last three coordinates and using Proposition 4.13, we get an infinite family of triples $\left(p_{n}, q_{n}, r_{n}\right)$ such that $\kappa\left(p_{1, n}, p_{2, n}, \ldots, p_{l(n), n}\right) \geq \kappa\left(p_{n}, q_{n}, r_{n}\right)$. As before, we may assume that $\kappa\left(p_{n}, q_{n}, r_{n}\right) \rightarrow \infty$, and hence, $\kappa\left(p_{1, n}, p_{2, n}, \ldots, p_{l(n), n}\right) \rightarrow \infty$.

To finish our argument we need to know that every positive integer can be realized as $\kappa$ of some Seifert homology sphere. Indeed, one can directly verify from Theorem 1.3 that $k(2,3,6 k+1)=k$. Hence, the proof is complete.

\section{Weakly Elliptic Brieskorn Spheres}

In this section we use our findings to characterize all weakly elliptic Brieskorn spheres $\Sigma(p, q, r)$ in terms of their defining integers $1<p<q<r$. We begin with introducing a new concept on numerical semigroups.

Definition 6.1. Let $G$ be a numerical semigroup and let $n_{0} \in \mathbb{N}-G$ be a positive integer. Then $G$ is said to alternate with respect to $n_{0}$, if for every $x, y \in G$ such that $x<y<n_{0}$, there exists $z \in G$ satisfying $x<n_{0}-z<y$.

Note that if $G$ is generated by a single element $a$, then $G$ alternates with respect to any $n_{0} \in$ $\mathbb{N}-G$. This notion gets more interesting if there are more than one generators. Clearly, in this case, there are only finitely many possibilities for $n_{0}$. 
Lemma 6.2. Let $G=G(a, b, c)$ be a numerical semigroup minimally generated by three relatively prime positive integers $a+1<b<c$, and let $n_{0}$ be a number from $\mathbb{N}-G$. Then $G$ alternates with respect to $n_{0}$ if and only if $a<n_{0}<b<c$.

Proof. $(\Leftarrow)$ Our claim is immediately proven once we replace $G(a, b, c)$ by $G(a)$.

$(\Rightarrow)$ Let $n_{0} \in \mathbb{N}-G$ be a positive integer with respect to which $G$ alternates. Clearly, if $n_{0}<a$, then there is nothing to prove. We proceed by induction on $n_{0}$, the base case being $n_{0}=a+1$. Notice that our claim is trivially true in the base case.

Assume now that if $n_{0}^{\prime}<n_{0}$ and $G$ is alternating with respect to $n_{0}^{\prime}$, then $a<n_{0}^{\prime}<b<c$. Suppose $x<y$ are from $G$ and they are the largest elements of $G$ that are less than $n_{0}$. Thus, there exists $z \in G$ such that $x<n_{0}-z<y<n_{0}$. It follows that $x+z<n_{0}<y+z<n_{0}+z$, hence $x+z=y$. Notice that $z$ has to be the smallest element $a$ of $G$, otherwise, for $w \in G$ with $w<z$ we see that $x<w+x<y$, contradicting with the maximality of $x$.

We claim that $G$ alternates with respect to $n_{0}^{\prime}=n_{0}-z$. Indeed, $n_{0}-z \notin G$ and if $u<v$ are two elements from $G$ such that $u<v<n_{0}-z$, then $u+z<v+z<n_{0}$, hence there exists $w \in G$ such that $u+z+w<n_{0}<v+z+w$. Our claim follows from this.

Now, by induction hypothesis we have that $a<n_{0}-z<b<c$. But $x<n_{0}-z$, so $x$ must be a multiple of $a$. Then $y=x+z$ is a multiple of $a$. If $n_{0}<b+z<y+z$, then $x<b<n_{0}$. Since $x$ is the second largest element of $G$ that is less than $n_{0}$, and since $b$ is not a multiple of $a$, we obtained a contradiction. Therefore, $y+z<b+z$, or $y<b$. This implies that $n_{0}<b$ and the proof is finished.

Corollary 6.3. Let $1<p<q<r$ be three relatively prime integers. Then the Brieskorn sphere $\Sigma(p, q, r)$ is weakly elliptic if and only if $N_{0}<p r$, where $N_{0}=p q r-p q-p r-q r$.

Proof. It follows from the discussion in Section 2 that $\Sigma=\Sigma(p, q, r)$ is weakly elliptic if and only if its difference function $\Delta_{\Sigma}$ alternates along its non-zero entries in the domain $\left[0, N_{0}\right]$. Interpreting in terms of the numerical semigroup $G_{\Sigma}=G(p q, p r, q r)$ of $\Sigma$, we see that if $p q<N_{0}<p r, \Delta_{\Sigma}$ alternates with respect to $N_{0}$ if and only if $G_{\Sigma}$ alternates with respect to $N_{0}$. On the other hand, if $0<N_{0}<p q$, there is nothing to prove, because there are only two non-zero values of $\Delta_{\Sigma}$ in $\left[0, N_{0}\right]$ and these are 1 and -1 .

Proof of Theorem 1.13. $(\Rightarrow)$ Let $\Sigma(p, q, r)$ be a weakly elliptic Brieskorn sphere. By Corollary 6.3, we know that $p q r-p q-p r-q r<p r$. Dividing by $p q r$, we obtain

$$
1-\frac{1}{r}-\frac{1}{q}-\frac{1}{p}<\frac{1}{q}
$$


Since $1<p<q<r$, it follows that $1-3 / p<1 / q$, or $1<1 / q+3 / p$, which implies $1<4 / p$. Thus, we conclude that $p<4$.

We proceed with the case $p=3$. Using (6.4) we see that $2 / 3-1 / r<2 / q$. Hence, if $r \geq 6$, then $2 / 3-1 / 6 \leq 2 / 3-1 / r<2 / q$. In other words, $1 / 2<2 / q$, or $q<4$, which is a contradiction. Therefore, $r<6$, hence the only possibility is that $q=4$ and $r=5$.

Next, we look at the case when $p=2$. Then we have

$$
\frac{1}{2}-\frac{1}{r}-\frac{1}{q}<\frac{1}{q}
$$

This inequality implies that $q<6$. There are two possibilities, $q=3$ and $q=5$. In the former case, we are done, already. For the latter, it follows from (6.5) that $r<10$. Obviously, the only two possibilities are $r=7$ and $r=9$.

$(\Leftarrow)$ It follows from the definition of weakly elliptic Brieskorn spheres and Table 1 that $\Sigma(2,5,7)$, $\Sigma(2,5,9), \Sigma(3,4,5), \Sigma(2,3,5), \Sigma(2,3,7)$, and $\Sigma(2,3,13)$ are weakly elliptic. Therefore, it is enough to show that $\Sigma(2,3, r), r>13$ is weakly elliptic.

Notice that any integer $r>13$ that is relatively prime to 2 and 3 has the form $r=6 k \pm 1$ for some $k \geq 3$. We proceed with the case that $r=6 k+1$. Then $N_{0}=6 k-5$. It follows that $6<N_{0}<2(6 k+1)<3(6 k+1)$, if $k \geq 3$. Therefore, by Corollary 6.3. $\Sigma(2,3,6 k+1)$ is weakly elliptic. In the next case that $r=6 k-1$, we have $N_{0}=6 k-7$. Similar to the previous case, $6<N_{0}<2(6 k-1)$, if $k \geq 3$. Therefore, $\Sigma(2,3,6 k-1)$ is weakly elliptic and the proof in the case of Brieskorn spheres is finished.

Finally, for more than three singular fibers, we observe that the statement and the proof of Corollary 6.3 is valid if $N_{0}<p_{1} p_{3} \cdots p_{l}$. However, an argument similar to "if" part of the proof of three singular fibers gives a contradiction to this inequality.

\section{Generating Function of $\tau$}

In this section we calculate the generating functions for the sequences $\tau(n)$ and $\Delta(n)$. Our main result shows that both generating functions are rational. For convenience we change our notation slightly. Let $\alpha=m / a=m_{1} / a_{1}, \beta=m_{2} / a_{2}$ and $\gamma=m_{3} / a_{3}$ be three rational numbers. Consider the integer valued function defined by the recurrence relation

$$
\tau(n+1)=\tau(n)+1+\left|e_{0}\right| n-\left\lceil\frac{n}{\alpha}\right\rceil-\left\lceil\frac{n}{\beta}\right\rceil-\left\lceil\frac{n}{\gamma}\right\rceil,
$$


and the initial condition $\tau(0)=0$.

Theorem 7.2. Let $\left(m_{1}, a_{1}\right),\left(m_{2}, a_{2}\right),\left(m_{3}, a_{3}\right)$ be three pairs of pairwise relatively prime positive integers, and let $\tau: \mathbb{N} \rightarrow \mathbb{Z}$ denote the $\tau$-function defined recursively as in (7.1). Then its generating series $F(x)=\sum_{n \geq 1} \tau(n) x^{n}$ is given by

$$
F(x)=\frac{x}{(1-x)^{2}}+\frac{\left|e_{0}\right| x^{2}}{(1-x)^{3}}-\frac{x^{2}}{(1-x)^{2}} \sum_{i=1}^{3} \frac{\left(1-x^{\left\lfloor m_{i} / a_{i}\right\rfloor a_{i}}\right)}{\left(1-x^{m_{i}}\right)\left(1-x^{\left\lfloor m_{i} / a_{i}\right\rfloor}\right)},
$$

where $\lfloor y\rfloor$ denotes the floor function.

Theorem 7.2 immediately implies Theorem 1.14. The proof of Theorem 7.2 occupies the rest of this subsection. The main component of the proof is the identification of the generating function $f(x)=\sum_{n \geq 0}\left\lceil\frac{n a}{m}\right\rceil x^{n}$ with a simple rational function. We achieve this in two steps.

Lemma 7.3. Let $D(x)$ denote the polynomial $D(x):=\sum_{i=1}^{m-1}\left\lceil\left\lceil\frac{i a}{m}\right\rceil x^{i}\right.$. Then

$$
f(x)=\frac{a x^{m}+D(x)(1-x)}{(1-x)\left(1-x^{m}\right)} .
$$

Proof. To compute $f(x)$ in a closed form we break it into congruence classes modulo $m$ (without worrying about convergence issues):

$$
f(x)=\sum_{n \equiv 0}\left\lceil\frac{n a}{m}\right\rceil x^{n}+\sum_{n \equiv 1}\left\lceil\frac{n a}{m}\right\rceil x^{n}+\cdots+\sum_{n \equiv m-1}\left\lceil\frac{n a}{m}\right\rceil x^{n},
$$

or

$$
f(x)=\sum_{l \geq 0}\left\lceil\frac{l m a}{m}\right\rceil x^{l m}+\sum_{l \geq 0}\left\lceil\frac{(l m+1) a}{m}\right\rceil x^{l m+1}+\cdots+\sum_{l \geq 0}\left\lceil\frac{(l m+m-1) a}{m}\right\rceil x^{l m+m-1} .
$$

Note that, for $i=1, \ldots, m-1$

$$
\sum_{l \geq 0}\left\lceil\frac{(l m+i) a}{m}\right\rceil x^{l m+i}=\sum_{l \geq 0}\left(l a+\left\lceil\frac{i a}{m}\right\rceil\right) x^{l m+i} .
$$

We separate the right hand side of (7.4) into two summations; $f(x)=A(x)+B(x)$, where

$$
A(x)=\sum_{i=0}^{m-1} \sum_{l \geq 0} a l x^{m l+i} \quad \text { and } \quad B(x)=\sum_{i=1}^{m-1} \sum_{l \geq 0}\left\lceil\frac{i a}{m}\right\rceil x^{m l+i} .
$$


It is easier to find a closed formula for $A(x)$;

$$
\begin{aligned}
A(x)=\sum_{i=0}^{m-1} \sum_{l \geq 0} a l x^{m l+i} & =\sum_{i=0}^{m-1} x^{i} \sum_{l \geq 0} a l x^{m l} \\
& =\frac{1-x^{m}}{1-x} a \sum_{l \geq 0} l\left(x^{m}\right)^{l}=\frac{1-x^{m}}{1-x} a x^{m} \frac{1}{\left(1-x^{m}\right)^{2}}=\frac{a x^{m}}{(1-x)\left(1-x^{m}\right)} .
\end{aligned}
$$

For $B(x)$ we have

$$
B(x)=\sum_{i=1}^{m-1} \sum_{l \geq 0}\left\lceil\frac{i a}{m}\right\rceil x^{m l+i}=\left(\sum_{i=1}^{m-1}\left\lceil\left\lceil\frac{i a}{m}\right\rceil x^{i}\right) \sum_{l \geq 0} x^{m l}=\left(\sum_{i=1}^{m-1}\left\lceil\frac{i a}{m}\right\rceil x^{i}\right) \frac{1}{1-x^{m}} .\right.
$$

Thus, if we define $D(x)$ as in hypothesis,

$$
f(x)=A(x)+B(x)=\frac{a x^{m}}{(1-x)\left(1-x^{m}\right)}+D(x) \frac{1}{1-x^{m}}=\frac{a x^{m}+D(x)(1-x)}{(1-x)\left(1-x^{m}\right)} .
$$

Lemma 7.5. Let $m=p a+q$ with $0 \leq q<a$ then $f(x)$ can be written as

$$
f(x)=\frac{x\left(1-x^{p a}\right)}{(1-x)\left(1-x^{m}\right)\left(1-x^{p}\right)}
$$

Proof. From Lemma 7.3,

$$
f(x)=\left(\sum_{i=0}^{m-1} c_{i} x^{i+1}\right) \frac{1}{(1-x)\left(1-x^{m}\right)}
$$

where

$$
c_{i}=\left\lceil\frac{(i+1) a}{m}\right\rceil-\left\lceil\frac{i a}{m}\right\rceil= \begin{cases}1 & \text { if } i \equiv 0(\bmod p) \\ 0 & \text { otherwise }\end{cases}
$$

Therefore

$$
f(x)=\frac{\sum_{j=1}^{a-1} x^{j p+1}}{(1-x)\left(1-x^{m}\right)}=\frac{x \sum_{j=1}^{a-1}\left(x^{p}\right)^{j}}{(1-x)\left(1-x^{m}\right)}=\frac{x\left(1-x^{p a}\right)}{(1-x)\left(1-x^{m}\right)\left(1-x^{p}\right)} .
$$

Proof of Theorem 7.2. Let $F(x)$ and $f_{i}(x)$ for $i=1,2,3$ denote the generating functions of $\tau(n)$ and $\left\lceil\frac{n a_{i}}{m_{i}}\right\rceil$, respectively. If we multiply both sides of the equation

$$
\tau(n+1)=\tau(n)+1+\left|e_{0}\right| n-\left\lceil\frac{n}{\alpha}\right\rceil-\left\lceil\frac{n}{\beta}\right\rceil-\left\lceil\frac{n}{\gamma}\right\rceil
$$


by $x^{n+1}$ and sum over $n \geq 0$, then we obtain

$$
F(x)=x F(x)+\sum_{n \geq 0} x^{n+1}+\sum_{n \geq 0}\left|e_{0}\right| n x^{n+1}-x\left(f_{1}(x)+f_{2}(x)+f_{3}(x)\right)
$$

Equivalently,

$$
F(x)=\frac{1}{1-x}\left(\frac{x}{1-x}+\frac{\left|e_{0}\right| x^{2}}{(1-x)^{2}}-x\left(f_{1}(x)+f_{2}(x)+f_{3}(x)\right)\right) .
$$

Therefore, the result follows from Lemma 7.5.

\subsection{Closed form of $\tau(n)$}

In this subsection we use the generating function given in Theorem 7.2 to find $\tau$ explicitly. See [9] for an alternative formula in terms of Dedekind sums.

Theorem 7.6. The unique solution to the recurrence defined in (7.1) is given by

$$
\begin{aligned}
\tau(n)=n & +\left|e_{0}\right|\left(\frac{n(n-1)}{2}\right) \\
& +\sum_{i=1}^{3} \sum_{k=0}^{a_{i}-1}\left(-\left(n-\left\lfloor m_{i} / a_{i}\right\rfloor k-1\right)+\frac{m_{i}}{2}\left\lfloor\frac{n-\left\lfloor m_{i} / a_{i}\right\rfloor k-1}{m_{i}}\right\rfloor\right)\left(\left\lfloor\frac{n-\left\lfloor m_{i} / a_{i}\right\rfloor k-1}{m_{i}}\right\rfloor+1\right) .
\end{aligned}
$$

Before starting the proof we first we state a useful lemma whose proof is omitted.

Lemma 7.7. If $g(x)=\sum_{n=0}^{\infty} c_{n} x^{n}$, then $\frac{x}{(1-x)^{2}} g(x)=\sum_{n=0}^{\infty}\left(\sum_{j=0}^{n}(n-j) c_{j}\right) x^{n}$.

Proof of Theorem 7.6. Let $p_{i}:=\left\lfloor m_{i} / a_{i}\right\rfloor$ for all $i=1,2,3$. By Theorem 7.2,

$$
\begin{aligned}
\sum_{n=0}^{\infty} \tau(n) x^{n} & =\frac{x}{(1-x)^{2}}\left(1+\frac{\left|e_{0}\right| x}{1-x}-\sum_{i=1}^{3} \sum_{k=0}^{a_{i}-1} \frac{x^{k p_{i}+1}}{1-x^{m_{i}}}\right) \\
& =\frac{x}{(1-x)^{2}}\left(1+\sum_{n=1}^{\infty}\left|e_{0}\right| x^{n}-\sum_{i=1}^{3} \sum_{k=0}^{a_{i}-1} \sum_{n=0}^{\infty} x^{m_{i} n+k p_{i}+1}\right) \\
& =\frac{x}{(1-x)^{2}}\left(\sum_{n=0}^{\infty} \xi(n) x^{n}-\sum_{n=0}^{\infty} \sum_{i=1}^{3} \sum_{k=0}^{a_{i}-1} \epsilon_{m_{i}}\left(n-k p_{i}-1\right) x^{n}\right) \\
& =\frac{x}{(1-x)^{2}}\left(\sum_{n=0}^{\infty} c_{n} x^{n}\right),
\end{aligned}
$$

where $c(n)=\xi(n)-\sum_{i=1}^{3} \sum_{k=0}^{a_{i}-1} \epsilon_{m_{i}}\left(n-k p_{i}-1\right)$, and 


$$
\epsilon_{m}(j)=\left\{\begin{array}{ll}
1 & \text { if } j \equiv 0(\bmod m) \\
0 & \text { otherwise }
\end{array} \quad \xi(n)= \begin{cases}0 & \text { if } n=0 \\
\left|e_{0}\right| & \text { otherwise }\end{cases}\right.
$$

By Lemma 7.7,

$$
\begin{aligned}
\tau(n) & =\sum_{j=0}^{n}(n-j) c_{j} \\
& =\sum_{j=0}^{n}(n-j) \xi(j)-\sum_{i=1}^{3} \sum_{k=0}^{a_{i}-1} \sum_{j=0}^{n}(n-j) \epsilon_{m_{i}}\left(j-k p_{i}-1\right) \\
& =n+\sum_{j=1}^{n}(n-j)\left|e_{0}\right|-\sum_{i=1}^{3} \sum_{k=0}^{a_{i}-1} \sum_{j=0}^{\left\lfloor\left(n-k p_{i}-1\right) / m_{i}\right\rfloor} n-\left(m_{i} j+k p_{i}+1\right) \\
& =n+\left|e_{0}\right| n^{2}-\frac{\left|e_{0}\right| n(n+1)}{2}-\sum_{i=1}^{3} \sum_{k=0}^{a_{i}-1}\left(\left(n-k p_{i}-1\right)-\frac{m_{i}}{2}\left(\left\lfloor\frac{n-k p_{i}-1}{m_{i}}\right\rfloor\right)\right)\left(\left\lfloor\frac{n-k p_{i}-1}{m_{i}}\right\rfloor+1\right) .
\end{aligned}
$$

Hence the proof is complete.

Acknowledgements. This collaboration has begun during 2012 Gökova Geometry-Topology Conference. Authors would like thank the organizers for providing such a stimulating atmosphere. Authors are grateful to Tye Lidman for helpful discussions. The second author is supported by the National Science Foundation Grant DMS-1065178, and by a Simons fellowship.

\section{References}

[1] Selman Akbulut and Çağrı Karakurt. Heegaard Floer homology of some Mazur type manifolds. arxiv:1204.3862.

[2] Selman Akbulut and John D. McCarthy. Casson's invariant for oriented homology 3-spheres, volume 36 of Mathematical Notes. Princeton University Press, Princeton, NJ, 1990. An exposition.

[3] Steven Boyer, Dale Rolfsen, and Bert Wiest. Orderable 3-manifold groups. Ann. Inst. Fourier (Grenoble), 55(1):243-288, 2005.

[4] Eaman Eftekhary. Seifert fibered homology spheres with trivial Heegaard Floer homology. arxiv:0909.3975. 
[5] Ronald Fintushel and Ronald J. Stern. Instanton homology of Seifert fibred homology three spheres. Proc. London Math. Soc. (3), 61(1):109-137, 1990.

[6] Paolo Lisca and András I. Stipsicz. Ozsváth-Szabó invariants and tight contact 3-manifolds. III. J. Symplectic Geom., 5(4):357-384, 2007.

[7] András Némethi. On the Heegaard Floer homology of $S_{-p / q}^{3}(K)$. arxiv:0410.570.

[8] András Némethi. On the Ozsváth-Szabó invariant of negative definite plumbed 3-manifolds. Geom. Topol., 9:991-1042, 2005.

[9] András Némethi and M. Borodzik. Heegaard Floer homologies for $(+1)$ surgeries on torus knots. arxiv:1105.5508.

[10] András Némethi and Liviu I. Nicolaescu. Seiberg-Witten invariants and surface singularities. Geom. Topol., 6:269-328 (electronic), 2002.

[11] Peter Ozsváth and Zoltán Szabó. Absolutely graded Floer homologies and intersection forms for four-manifolds with boundary. Adv. Math., 173(2):179-261, 2003.

[12] Peter Ozsváth and Zoltán Szabó. On the Floer homology of plumbed three-manifolds. Geom. Topol., 7:185-224 (electronic), 2003.

[13] Peter Ozsváth and Zoltán Szabó. Holomorphic disks and three-manifold invariants: properties and applications. Ann. of Math. (2), 159(3):1159-1245, 2004.

[14] Peter Ozsváth and Zoltán Szabó. Holomorphic disks and topological invariants for closed three-manifolds. Ann. of Math. (2), 159(3):1027-1158, 2004.

[15] Raif Rustamov. On plumbed l-spaces. arxiv:math/0505349.

[16] Eamonn Tweedy. Heegaard Floer homology and several families of Brieskorn spheres. arxiv:1206.2558. 